\title{
Multiples of Divisors
}

\section{ERNST SNAPPER}

Introduction. The main purpose of this paper is to prove Theorem 9.1 and to prepare for the study of the polynomials which occur in it. The investigation of these polynomials has been carried out in our paper Polynomials Associated With Divisors, to be published in the Journal of Mathematics and Mechanics, 9 (1960).

Let $X$ be an irreducible, normal, projective variety defined over an arbitrary groundfield. The sheaf of local rings of $X$ is denoted by $L$, and the sheaf of the locally non-vanishing rational functions of $X$ by $L_{0}$. The group structure in $L_{0}$ is given by the multiplication, and the multiplicative cohomology group $H^{1}\left(X, L_{0}\right)$ is isomorphic with the additive group of those divisor classes of $X$, with respect to linear equivalence, whose divisors are locally linearly equivalent to zero. All these terms are clear, at least intuitively, and they have been given rigorous definitions in the course of this paper.

We now say a word about the interpretation of Theorem 9.1 in terms of divisors. If $D$ is a divisor of $X$ and $F$ is an algebraic sheaf of $X$, we denote the sheaf $L(D) \otimes_{L} F$ by $F(D)$, where the sheaf $L(D)$ is defined as usual. (See, for example, [6] or [11] for $L(D)$; square brackets refer to the references.) Let $D_{1}, \cdots, D_{n}$ be divisors of $X$ which are all locally linearly equivalent to zero and let $F$ be an algebraic coherent sheaf of $X$. The ring of the rational integers is denoted by $Z$ and "rational polynomial" means "polynomial whose coefficients are rational numbers." By studying the functorial properties of the elements of $H^{1}\left(X, L_{0}\right)$, we are able to show in Theorem 9.1 that the Euler-Poincaré characteristic $\chi\left(X, F\left(m_{1} D_{1}+\cdots+m_{n} D_{n}\right)\right)$ is a rational polynomial in $m_{1}, \cdots$, $m_{n}$ for all $m_{1}, \cdots, m_{n} \varepsilon Z$; furthermore, the degree of this polynomial does not exceed the dimension of the support of $F$. (The associated integral polynomials have been studied in Polynomials Associated With Divisors.) Example 10.1 shows that this degree may be less than the dimension of the support of $F$; example 10.2 shows that if the divisor $D$ of $X$ is not locally linearly equivalent to zero, then $\chi(X, L(m D))$ may not be a rational polynomial in $m$. This last example was essentially given to the author by Professor ZARIsKI in 1954.

The elements of $H^{1}\left(X, L_{0}\right)$ are called line classes because they correspond to the classes of isomorphic line bundles of section 3 of [4]. Theorem 9.1 has been formulated in terms of line classes and holds with this formulation for all pro-

This research was supported in part by National Science Foundation Grant No. G5883. 
jective varieties, including the reducible and not normal ones. We are convinced that this theorem is correct for all complete algebraic varieties, including the non-projective ones. We were not able to prove this because we could prove Corollary 8.1 only in the projective case. Except for the use of this corollary in case 3c of section 9, our proof of Theorem 9.1 is valid for all complete algebraic varieties. In this connection we point out that if $F$ is an algebraic coherent sheaf of a complete algebraic variety $X$, then $\chi(X, F)$ is well defined because of Theorem 2 of [2] and of the finiteness theorem of section 5 of [10]. The method we used to prove Theorem 9.1 is what is called in [11] "the inductive method." The general sheaf-theoretic tools which are necessary for the application of this inductive method have been constructed in [2]. This is why [2] is so important, well over and above the very fine theorems which are actually derived there.

In the first seven sections we develop the functorial theory of the line classes of not necessarily projective, algebraic varieties. Whenever we assume that our variety is complete, the only reason for this is that we want certain cohomology groups to be finite dimensional vector spaces over the groundfield. The assumption of completeness can be avoided wherever this finite dimensionality plays no role. The symbol $\simeq$ is used to denote all types of isomorphisms, and the type has not been specified unless there is danger of confusion. When we refer to, say, "Proposition 5.2" without further designation, we are referring to the second proposition of section 5 of the present paper.

1. The fibre classes of a sheaf of rings. Let $(X, A, p)$ be a sheaf of rings. Here $X$ is the base space, $A$ the sheaf space and $p$ the projection of $A$ onto $X$. See [1], section 6 , for sheaves of rings. The topology of $X$ is arbitrary and, if $x \varepsilon X$, the ring $A_{x}$ is not necessarily commutative. As in [1], we assume that $A_{x}$ has a unit element $1_{x}$ and that the mapping $x \rightarrow 1_{x}$ is a section of $A$ over $X$. The (multiplicative) group of units of $A_{x}$ is denoted by $G_{x}$ and $G=\cup G_{x}$, where $x$ runs through $X$. We consider $G$ as a subspace of the topological space $A$ and denote the restriction of $p$ to $G$ by $p_{0}$.

Proposition 1.1. $\left(X, G, p_{0}\right)$ is a sheaf of (multiplicative) groups over $X$ as base space. If $Y$ is a subset of $X$, the group of sections of $G$ over $Y$ is the group of units of the ring of sections of $A$ over $Y$.

Proof. Let $x \varepsilon X$ and $g \varepsilon G_{x}$. Select an open neighborhood $U$ of $x$ such that there exists a section $s$ of $A$ over $U$ whose value at $x$ is $g$. Since $A$ is of finite type (see [1], section 15), we conclude from Proposition 1 of section 12 of [1] that $U$ contains a neighborhood $V$ of $x$ such that $s(V) \subset G$. This proves that $G$ is an open subset of $A$ and hence that $p_{0}$ is a local homeomorphism from $G$ onto $X$. Consider now a subset $Y$ of $X$ and a section $s$ of $A$ over $Y$ with the property that $s(Y) \subset G$. Then, for each $x \varepsilon Y,(s(x))^{-1}$ is a well defined element of $A_{x}$ and the mapping $s^{-1}: Y \rightarrow A$, defined by $s^{-1}(x)=(s(x))^{-1}$, is a section of $A$ over $Y$; this is an easy consequence of the continuity of the mapping $x \rightarrow 1_{x}$, 
mentioned earlier. The remainder of Proposition 1.1 follows immediately from the fact that $s^{-1}$ is a section.

We call $G$ the sheaf of units of $A$. The first cohomology set $H^{1}(X, G)$ of $X$ with values in $G$ is well defined and is a multiplicative, commutative group if $A$, and hence $G$, is commutative; see [3], section 5.1.

Definition 1.1. A fibre class of $A$ is an element of $H^{1}(X, G)$.

The set of fibre classes of $A$ is in $(1,1)$-correspondence with the set of "isomorphism classes of fibre spaces with structure sheaf $G$ " in the sense of [3]. This explains our term "fibre class."

2. Fibre classes as functors. If $F$ is a sheaf of any kind of algebraic structure, the algebraic structure of the sections of $F$ over a subset $Y$ of the base space will be denoted by $S(Y, F)$, and the restriction of $F$ to $Y$ by $F(Y)$; see [1], section 4, for the precise definition of $F(Y)$.

Let $(X, A, p)$ and $\left(X, G, p_{0}\right)$ be as in the previous section. We assume from now on that $A$ is a sheaf of commutative rings, whence the fibre classes of $A$ form the multiplicative, commutative group $H^{1}(X, G)$. Let $f$ be a fibre class of $A$ and let $\mathfrak{U}=\left\{U_{i}, i \varepsilon I\right\}$ be an indexed, open covering of $X$ which admits a 1-cocycle $b$ with values in $G$ which is a representative of $f$. This implies that if $i, j \varepsilon I$, then $b(i, j) \varepsilon S\left(U_{i} \cap U_{j}, G\right)$ and if $i, j, h \varepsilon I$, then $b(i, h)=b(i, j) b(j, h)$ over $U_{i} \cap U_{i} \cap U_{h}$. Consider a sheaf $F$ of $A$-modules; see [1], section 6 , for the notion of a sheaf of $A$-modules. For each $i \varepsilon I$, the sheaf of $A\left(U_{i}\right)$-modules $F\left(U_{i}\right)=F_{i}$ is well defined. For each pair $i, j \varepsilon I$, we have the $A\left(U_{i} \cap U_{i}\right)$-isomorphism $t_{i j}: F_{i}\left(U_{i} \cap U_{i}\right) \rightarrow F_{i}\left(U_{i} \cap U_{i}\right)$ which is defined by $t_{i j}(a)=$ $(b(i, j)(x))(a)$ if $x \boldsymbol{\varepsilon} U_{i} \cap U_{j}$ and $a \varepsilon\left(F_{i}\right)_{x}$. The relation $t_{i h}=t_{i j} t_{j h}$ over $U_{i} \cap U_{j} \cap U_{h}$ follows from the corresponding relation for $b$, mentioned above. We know from [1], section 4, Proposition 4, that there exists an $A$-sheaf $K$ ( $A$-sheaf means sheaf of $A$-modules) and, for each $i \varepsilon I$, an $A\left(U_{i}\right)$-isomorphism $u_{i}: K\left(U_{i}\right) \rightarrow$ $F\left(U_{i}\right)$ such that $t_{i j} u_{i}=u_{i}$ over $U_{i} \cap U_{i}$. Furthermore, the system $\left\{K, u_{i}\right\}$ is uniquely determined, except for isomorphisms, by $F$ and $b$. Let now $\mathfrak{B}=$ $\left\{V_{i}, j \varepsilon J\right\}$ be some other indexed, open covering of $X$ which admits a 1-cocycle $c$ with values in $G$ which represents $f$. We construct the system $\left\{L, v_{i}\right\}$, consisting of an $A$-sheaf $L$ and $A\left(V_{i}\right)$-isomorphisms $v_{i}: L\left(V_{i}\right) \rightarrow F\left(V_{i}\right)$, by means of $F$ and $c$ in the same way as the system $\left\{K, u_{i}\right\}$ was constructed by means of $F$ and $b$.

Proposition 2.1. $K$ and $L$ are $A$-isomorphic.

Proof. Case $1 . \mathfrak{B}$ is a refinement of $\mathfrak{U}$, the mapping $s: J \rightarrow I$ is such that $V_{j} \subset U_{s(i)}$ for all $j \varepsilon J$, and $c=s^{*}(b)$; i.e., if $j_{0}, j_{1} \varepsilon J$, then $c\left(j_{0}, j_{1}\right)=$ $r\left(b\left(s\left(j_{0}\right), s\left(j_{1}\right)\right)\right)$, where $r$ denotes the restriction map from $U_{s\left(i_{0}\right)} \cap U_{s\left(i_{1}\right)}$ to $V_{i_{0}} \cap V_{i_{1}}$. We now describe an $A$-isomorphism $w: L \rightarrow K$ for the special case 
that $c=s^{*}(b)$. Accordingly, select $g \varepsilon L_{x}$ for some $x \varepsilon X$. There exists some $j \varepsilon J$ such that $x \varepsilon V_{i}$, and we define $w(g)=u_{i}^{-1} v_{i}(g)$, where $i=s(j)$. We verify easily that $w(g)$ is independent of the choice of $j$ and that $w: L \rightarrow K$ is an $A$ isomorphism.

Case 2. $\mathfrak{U}=\mathfrak{B}$, and $b$ and $c$ are cohomologous. This means that there exists a 0 -cochain $e$ of $\mathfrak{U}$ with values in $G$ such that $b^{-1} c$ is the coboundary of $e$; i.e., if $i \varepsilon I$, then $e(i) \varepsilon S\left(U_{i}, G\right)$ and if $i, j \varepsilon I$, then $e(j) b(i, j)=e(i) c(i, j)$ over $U_{i} \cap U_{i}$. The $A$-isomorphism $w: L \rightarrow K$ can now be described as follows. If $g \varepsilon L_{x}$, select $i_{\varepsilon} I$ such that $x \& U_{i}$. We then define $w(g)=e_{i}(x) u_{i}^{-1} v_{i}(g)$. Again, we verify easily that $w(g)$ does not depend on the choice of $i$ and that $w: L \rightarrow K$ is an $A$-isomorphism.

Case 3. $\mathfrak{A}, \mathfrak{B}, b, c$ are arbitrary. Since $b$ and $c$ both represent $f$, there exists a common refinement $\mathfrak{B}$ of $\mathfrak{U}$ and $\mathfrak{B}$ and cohomologous 1 -cocycles $b^{\prime}, c^{\prime}$ of $\mathfrak{W}$ with values in $G$ such that both pairs $\left(b, b^{\prime}\right)$ and $\left(c, c^{\prime}\right)$ fall under case 1 . Hence if $K^{\prime}, L^{\prime}$ are the $A$-sheaves which arise from $F$ and, respectively, $b^{\prime}$ and $c^{\prime}$, then $K \simeq K^{\prime}$ and $L \simeq L^{\prime}$. According to case $2, K^{\prime} \simeq L^{\prime}$, which completes the proof of Proposition 2.1.

Let $[F]$ denote the class of $A$-sheaves which are $A$-isomorphic with $F$. Proposition 2.1 shows that a class $f([F])$ of isomorphic $A$-sheaves is well defined. In order to find an $A$-sheaf $K \varepsilon f([F])$, we simply transform any $A$-sheaf which belongs to $[F]$ by means of any 1-cocycle $b$ which represents $f$. We will write $f(F)$ instead of $f([F])$, thereby ignoring in our notation the fact that $f(F)$ is determined only up to isomorphisms.

Proposition 2.2. A fibre class $f$ of $A$ can be considered as a covariant, exact functor from the category of the (classes of $A$-isomorphic) $A$-sheaves and (classes of equivalent) A-homomorphisms to the same category. If $F$ is an A-sheaf, $f(F)$ is locally isomorphic with $F$ and hence, if $F$ is of finite type or coherent, the same is true for $f(F)$. If $g$ is a second fibre class, $(f g)(F) \simeq f(g(F))$. If $M$ is a second $A$-sheaf, $f(F) \otimes_{A} g(M) \simeq f g\left(F \bigotimes_{A} M\right)$. Here $f g$ denotes the product in the group $H^{1}(X, G)$ and $\simeq$ denotes $A$-isomorphism.

Proof. Let $F$ and $F^{\prime}$ be two $A$-sheaves and $a: F \rightarrow F^{\prime}$ an $A$-homomorphism. In order to construct the $A$-homomorphism $f(a): f(F) \rightarrow f\left(F^{\prime}\right)$, select an indexed, open covering $\mathfrak{U}=\left\{U_{i}, I\right\}$ of $X$ which admits a 1-cocycle $b$ which represents $f$. Let $\left\{f(F), u_{i}\right\}$ and $\left\{f\left(F^{\prime}\right), v_{i}\right\}$ be the systems which result from transforming $F$ and $F^{\prime}$ by $b$. If $g \varepsilon(f(F))_{x}$, select $i \varepsilon I$ such that $x \varepsilon U_{i}$. We define $(f(a))(g)=$ $v_{i}^{-1} a u_{i}(g)$, and verify easily that $(f(a))(g)$ does not depend on the choice of $i$ and that $f(a): f(F) \rightarrow f\left(F^{\prime}\right)$ is an $A$-homomorphism. It is now clear that $f$ may be considered as a covariant functor. If $F \stackrel{a}{\rightarrow} F^{\prime} \stackrel{a}{\rightarrow} F^{\prime \prime}$ is a sequence of $A$-sheaves and $A$-homomorphisms, we have, for each $i \varepsilon I$, the commutative diagram

$$
\begin{array}{ccccc}
F\left(U_{i}\right) & \stackrel{a}{\rightarrow} & F^{\prime}\left(U_{i}\right) & \stackrel{a^{\prime}}{\rightarrow} & F^{\prime \prime}\left(U_{i}\right) \\
\uparrow & & \uparrow & & \uparrow \\
f\left(F^{\prime}\right)\left(U_{i}\right) & \underset{f(a)}{\rightarrow} f\left(F^{\prime}\right)\left(U_{i}\right) \underset{f\left(a^{\prime}\right)}{\rightarrow} & f\left(F^{\prime \prime}\right)\left(U_{i}\right)
\end{array}
$$


The vertical arrows are the usual $A\left(U_{i}\right)$-isomorphisms, and hence the lower sequence is exact as soon as the upper sequence is exact. This shows that the functor to which $f$ gives rise is exact. We have now proved the first sentence of Proposition 2.2, while the second sentence requires no proof. (See [1], section 12, for sheaves of finite type and coherent sheaves.) Let now $g$ be a second fibre class. We may assume that our $\mathfrak{U}$ was so fine that $\mathfrak{U}$ also admits a 1-cocycle $c$ which represents $g$, whence $b c$ represents $f g$. Let the systems $\left\{g(F), v_{i}\right\}$ and $\left\{f(g(F)), u_{i}\right\}$ result from first transforming the $A$-sheaf $F$ by $c$ and then transforming $g(F)$ by $b$. In order to show that $(f g)(F) \simeq f(g(F))$, all we have to show is that the system $\left\{f(g(F)), v_{i} u_{i}\right\}$ may be regarded as resulting from transforming $F$ by $b c$. Accordingly, we only have to prove that if $i, j \varepsilon I$, then $v_{j} u_{i}=t_{i i} v_{i} u_{i}$ over $U_{i} \cap U_{i}$, where $t_{j i}$ consists of multiplying by $b(j, i) c(j, i)$. This, however, follows immediately from the fact that

$$
b(j, i) c(j, i) v_{i} u_{i}=\left(c(j, i) v_{i}\right)\left(b(j, i) u_{i}\right)=v_{j} u_{i}
$$

over $U_{i} \cap U_{j}$. Finally, consider the $A$-sheaf $M$ and the systems $\left\{f(F), u_{i}\right\}$ and $\left\{g(M), v_{i}\right\}$ which are obtained by transforming $F$ and $M$ by, respectively, $b$ and $c$. Then $u_{i} \otimes v_{i}:\left(f(F) \otimes_{A} g(M)\right)\left(U_{i}\right) \rightarrow\left(F \otimes_{A} M\right)\left(U_{i}\right)$ is an $A\left(U_{i}\right)$-isomorphism for each $i \varepsilon I$, so that we can consider the system $\left\{f(F) \bigotimes_{A} g(M)\right.$, $\left.u_{i} \otimes v_{i}\right\}$. In order to show that $f(F) \otimes_{A} g(M) \simeq f g\left(F \bigotimes_{A} M\right)$, we have to show that this system may be regarded as resulting from transforming $F \otimes_{A} M$ by means of $b c$; i.e., we have to prove that if $i, j \varepsilon I$, then $u_{i} \otimes v_{i}=t_{i i}\left(u_{i} \otimes v_{i}\right)$ over $U_{i} \cap U_{i}$, where $t_{i i}$ consists of multiplying by $b(j, i) c(j, i)$. This, however, follows from the fact that

$$
b(j, i) c(j, i)\left(u_{i} \otimes v_{i}\right)=b(j, i) u_{i} \otimes c(j, i) v_{i}=u_{i} \otimes v_{i}
$$

over $U_{i} \cap U_{j}$.

Let $Y$ be a subset of $X$. Evidently, the sheaf of units of $A(Y)$ is $G(Y)$, and hence to every fibre class $f$ of $A$ corresponds a unique fibre class $g$ of $A(Y)$. Precisely, let the indexed, open covering $\left\{U_{i}, i \varepsilon I\right\}$ of $X$ admit a 1-cocycle $b$ with values in $G$ which is a representative of $f$. The induced covering $\left\{U_{i} \cap Y, i \varepsilon I\right\}$ of $Y$ then carries the 1-cocycle $c$ with values in $G(Y)$ which is defined by $c(i, j)=r(b(i, j))$ if $i, j \varepsilon I$, where $r$ denotes the restriction map from $U_{i} \cap U_{i}$ to $U_{i} \cap U_{i} \cap Y$. This $c$ is a representative of $g$; see [1], section 26. Consequently, for every $A$-sheaf $F$, we can consider the two $A(Y)$-sheaves $(f(F))(Y)$ and $g(F(Y))$.

Proposition 2.3. The sheaves $(f(F))(Y)$ and $g(F(Y))$ are $A(Y)$-isomorphic.

Proof. Let the system $\left\{K, u_{i}\right\}$ be constructed by means of $F$ and $b$. We denote by $v_{i}$ the restriction of the $A\left(U_{i}\right)$-isomorphism $u_{i}: K\left(U_{i}\right) \rightarrow F\left(U_{i}\right)$ to $U_{i} \cap Y$. We prove easily that the system $\left\{K(Y), v_{i}\right\}$ may be regarded as the transform of $F(Y)$ by means of $c$, whence $K(Y) \simeq g(F(Y))$.

We now turn to "change of rings." Let $B$ be a second sheaf of commutative rings over $X$ as base space; we denote the sheaf of units of $B$ by $P$. Let $h: A \rightarrow B$ be a unitary sheaf homomorphism; the term "unitary" indicates that, for each 
$x \varepsilon X$, the ring homomorphism $h_{x}: A_{x} \rightarrow B_{x}$ maps the unit element of $A_{x}$ on the unit element of $B_{x}$. It follows that $h_{x}$ maps the group of units $G_{x}$ of $A_{x}$ homomorphically into the group of units $P_{x}$ of $B_{x}$, whence we have a sheaf homomorphism $h^{\prime}: G \rightarrow P$ and a group homomorphism $h^{*}: H^{1}(X, G) \rightarrow H^{1}(X, P)$.

Now let $f$ be a fibre class of $A$ and $g=h^{*}(f)$ the induced fibre class of $B$. If $F$ is a $B$-sheaf, we can consider $F$ as an $A$-sheaf by the usual "change of rings" technique; see [1], section 16 . In particular, the $B$-sheaf $g(F)$ can be considered as an $A$-sheaf.

Proposition 2.4. The A-sheaves $g(F)$ and $f(F)$ are A-isomorphic.

Proof. Let $\mathfrak{U}=\left\{U_{i}, I\right\}$ be an indexed, open covering of $X$ which admits a 1-cocycle $b$ with values in $G$ which represents $f$. A 1-cocycle $c$ of $\mathfrak{u}$ with values in $P$ which represents $g$ is defined by $c(i, j)=h(b(i, j))$ if $i, j \varepsilon I$. Construct a system $\left\{K, u_{i}\right\}$ by means of $c$ and the $B$-sheaf $F$. Then $K$ is a $B$-sheaf and each $u_{i}: K\left(U_{i}\right) \rightarrow F\left(U_{i}\right)$ is a $B\left(U_{i}\right)$-isomorphism. If we consider $K$ and $F$ as $A$ sheaves, $u_{i}$ becomes an $A\left(U_{i}\right)$-isomorphism, and the system $\left\{K, u_{i}\right\}$ may then be regarded as having been obtained by transforming the $A$-sheaf $F$ by means of $b$.

We continue our assumptions about $h: A \rightarrow B$ and $G, P, f, g=h^{*}(f)$. We assume, furthermore, that $Y$ is a closed subset of $X$ and that $F$ is a $B$-sheaf whose support is contained in $Y$. (See [1], section 81, for supports of sheaves.) We denote by $e$ the fibre class of $B(Y)$ which arises from $g$. The usual notation $H^{t}(X, F)$ for the $t^{\text {th }}$ cohomology group of $X$ with values in $F$ will be used.

Proposition 2.5. $H^{t}(X, f(F)) \simeq H^{t}(Y, e(F(Y)))$ for all $t \geqq 0$. Here $\simeq$ denotes group isomorphism.

Proof.

$$
\begin{aligned}
H^{t}(X, f(F)) & \simeq H^{t}(X, g(F)) \quad \text { follows from Proposition 2.4. } \\
H^{t}(Y, e(F(Y))) & \simeq H^{t}(Y,(g(F))(Y)) \text { follows from Proposition 2.3. } \\
H^{t}(X, g(F)) & \simeq H^{t}(Y,(g(F))(Y)) \text { follows from [1], section2 6, Proposition 8, }
\end{aligned}
$$
since $Y$ is closed and the support of $g(F)$ is contained in $Y$.

In the applications which we will make of Proposition 2.5, the cohomology groups will be vector spaces over a field $k$. It will be obvious that in these situations the symbol $\simeq$ in Proposition 2.5 may be regarded as a $k$-linear isomorphism.

3. The sections of $f(A)$ over $X$. Let $(X, A, p)$ and $\left(X, G, p_{0}\right)$ be as in the previous section; we repeat that $A$ is a sheaf of commutative rings. Let $f$ be a fibre class of $A$ and $F$ an $A$-sheaf. A section $s \varepsilon S(X, f(A))$ determines an $A$ homomorphism $t: F \rightarrow f(A) \bigotimes_{A} F$ which is defined by $t(g)=s(x) \otimes g$, if $x \varepsilon X$ and $g \& F_{x}$, where the tensor product is relative to $A_{x}$. If, in Proposition 2.2, we use for $g$ the identity element of $H^{1}(X, G)$, we see that $f(A) \otimes_{A} F \simeq f\left(A \otimes_{A} F\right)$ $\simeq f(F)$. Consequently, $s$ gives rise to an $A$-homomorphism $w: F \rightarrow f(F)$. Of course, $w$ is not uniquely determined by $s$, since the $A$-isomorphism $j: f(A) \otimes_{A} F \rightarrow f(F)$ depends on the choice of representative of $f$. Precisely, 
let $\mathfrak{U}=\left\{U_{i}, i \varepsilon I\right\}$ be an indexed, open covering of $X$ which admits a representative $b$ of $f$. Let the system $\left\{f(A), u_{i}\right\}$ be obtained by means of $A$ and $b$, and let the system $\left\{f(F), v_{i}\right\}$ be obtained by means of $F$ and $b$. Then for $j$ we may use the $A$-isomorphism which is defined by $j(h \otimes g)=u_{i}(h) v_{i}^{-1}(g)$ if $x \varepsilon U_{i}$ and $h \varepsilon(f(A))_{x}$ and $g \varepsilon F_{x}$. With this definition of $j$ we obtain

$$
w(g)=j t(g)=j(s(x) \otimes g)=u_{i}(s(x)) v_{i}^{-1}(g) .
$$

This leads us to consider the system $\left\{s_{i}, i \varepsilon I\right\}$, where $s_{i}=u_{i}\left(r_{i}(s)\right)$ is a section of $A$ over $U_{i}$; here $r_{i}(s)$ is the restriction of $s$ to $U_{i}$, whence $u_{i}(s(x))=s_{i}(x)$. Clearly, $s_{i}=b(j, i) s_{i}$ over $U_{i} \cap U_{j}$ for all $i, j \varepsilon I$, and all the sections of $f(A)$ over $X$ are determined by such systems of sections of $A$ over the various $U_{i}$. We collect these facts in the following proposition.

Proposition 3.1. Let $b$ \& $H^{1}(\mathfrak{U}, G)$ represent $f$ and let the system $\left\{f(F), v_{i}\right\}$ be obtained by means of $F$ and $b$. Let $\left\{s_{i}, i \varepsilon I\right\}$, where $s_{i} \varepsilon S\left(U_{i}, A\right)$, be any system with the property that $s_{j}=b(j, i) s_{i}$ over $U_{i} \cap U_{j}$ for all $i, j \varepsilon I$. Then the mapping which is defined by $w(g)=s_{i}(x) v_{i}^{-1}(g)$, if $x \varepsilon U_{i}$ and $g \varepsilon F_{x}$, is a well defined $A$-homomorphism $w: F \rightarrow f(F)$.

We now study the supports of the kernel and the cokernel of $w: F \rightarrow f(F)$; we write "Sup" for support.

Proposition 3.2. Let $x \varepsilon U_{i}$. Then $x \varepsilon$ Sup (coker $(w)$ ) if and only if $s_{i}(x) F_{x}$ $\neq F_{x} ;$ and $x \varepsilon \operatorname{Sup}(\operatorname{ker}(w))$ if and only if $s_{i}(x) g=0$ for some nonzero $g \varepsilon F_{x}$.

Proof. Since (coker $(w))_{x}=(f(F))_{x} / w\left(F_{x}\right)$ and $w\left(F_{x}\right)=s_{i}(x) v_{i}^{-1}\left(F_{x}\right)=$ $s_{i}(x)(f(F))_{x}$, we conclude that $x \varepsilon$ Sup (coker $\left.(w)\right)$ if and only if $s_{i}(x)(f(F))_{x} \neq$ $(f(F))_{x}$. This inequality is equivalent to $s_{i}(x) F_{x} \neq F_{x}$ because $(f(F))_{x}$ and $F_{x}$ are $A_{x}$-isomorphic. Furthermore, since the restriction of $v_{i}^{-1}$ to $F_{x}$ is an $A_{x^{-}}$ isomorphism, an element $g$ of $F_{x}$ belongs to ker $(w)$ if and only if $s_{i}(x) g=0$. It follows that Sup $(\operatorname{ker}(w))$ is as stated in Proposition 3.2.

We saw at the beginning of the last proof that if $x \varepsilon U_{i}$, then $(\operatorname{Im}(w))_{x}=$ $s_{i}(x)(f(F))_{x}$ where Im means "image." If we use the $A$-sheaf $f^{-1}(A)$ as $F$, we may use $A$ as $f\left(f^{-1}(A)\right)$ according to Proposition 2.2. Consequently, the system $\left\{s_{i}, i \varepsilon I\right\}$ gives rise to an $A$-homomorphism $w^{\prime}: f^{-1}(A) \rightarrow A$ and $\left(\operatorname{Im}\left(w^{\prime}\right)\right)_{x}=$ $s_{i}(x) A_{x}$ for $x \varepsilon U_{i}$. This explains the meaning of the sheaf $B$ of principal ideals of $A$, where $B_{x}=s_{i}(x) A_{x}$ for $x \varepsilon U_{i}$ and $i \varepsilon I$.

4. Line classes of algebraic varieties. Let $X$ be an algebraic variety, defined over an arbitrary groundfield $k$, and let $L$ be its sheaf of local rings; see [1], section 34 , for the notion of an algebraic variety. We need a little preparation concerning coherent sheaves of ideals of $X$.

Let $C$ be a coherent sheaf of ideals of $X$. As in section 2 of [2], we define the variety $\operatorname{Var}(C) \subset X$ of $C$ as follows: $x \varepsilon \operatorname{Var}(C)$ if $C_{x} \neq L_{x}$. We know from this reference that $\operatorname{Var}(C)$ is a closed subset of $X$. Consequently, if we extend the sheaf of local rings of $\operatorname{Var}(C)$ by zero over all of $X$, we obtain an algebraic, 
coherent sheaf $F$ of $X$. We denote the annihilator of $F$ by a, and we know from [1], section 39, that $\mathfrak{a}$ is a coherent sheaf of ideals of $X$. (The radical of an ideal $\mathfrak{b}$ of a ring is the ideal which consists of the ring elements of which a positive power belongs to $\mathfrak{b}$. If $\operatorname{Var}(C)=\phi$, clearly $F=0$ and $\mathfrak{a}=L$.)

Proposition 4.1. $\mathfrak{a}_{x}$ is the radical of the ideal $C_{x}$. The quotient sheaf $L / C$, restricted to $\operatorname{Var}(C)$, is an algebraic coherent sheaf over $\operatorname{Var}(C)$ if and only if $C=$ a. In this case, $L / C$ may be regarded as the sheaf of local rings of $\operatorname{Var}(C)$.

Proof. We know from [1], section 39, that there exists an exact sequence of $L$-homomorphisms $0 \rightarrow \mathfrak{a} \rightarrow L \rightarrow F \rightarrow 0$, whence the variety of $\mathfrak{a}$ is equal to the support of $F$, i.e. to $\operatorname{Var}(C)$. It now follows from [2], lemma 8 , that $\mathfrak{a}_{x}$ and $C_{x}$ have the same radical. Furthermore, $L_{x} / \mathfrak{a}_{x} \simeq F_{x}$, whence $\mathfrak{a}_{x}=\operatorname{radical}\left(\mathfrak{a}_{x}\right)$ because a local ring $F_{x}$ of an algebraic variety (even if that variety is reducible) does not possess nonzero nilpotent elements (see [1], section 48). We also know from [1], section 39, that $L / C$ is an algebraic coherent sheaf over $\operatorname{Var}(C)$ if and only if $C \supset \mathfrak{a}$ which, since $\mathfrak{a} \supset C$, is equivalent to $C=\mathfrak{a}$. Finally, the above exact sequence shows that if $C=\mathfrak{a}$, then $F \simeq L / C$.

We denote the sheaf of units of $L$ by $L_{0}$ and call the fibre classes of $L$ the line classes of $X$. This $L_{0}$ is "the sheaf of the locally nonvanishing rational functions of $X$ " of the introduction. We would have preferred to say line bundle instead of line class, but have decided on the present terminology in order that our notion of line class will correspond to that of "a class of isomorphic line bundles" of section 3 of [4]. Whenever we write $\mathfrak{U}$ or $\mathfrak{U}=\left\{U_{i}, I\right\}$, it is always understood that $\mathfrak{U}$ is an indexed, open covering of $X$.

We assume for the remainder of this section that $X$ is irreducible, and denote by $E$ the field of rational functions of $X$. To each $x \in X$ is associated the local ring $Q_{x} \subset E$ of $x$ with maximal ideal $\mathfrak{m}_{x}$, and we have a ring isomorphism $j_{x}: L_{x} \rightarrow Q_{x}$; see [1], section 36 . We know from the same reference that we may consider $L$ as an algebraic subsheaf of the constant, algebraic sheaf $E^{\prime}$ associated with $E$ and $X$. ("Algebraic sheaf" means $L$-sheaf.)

Definition 4.1. Let $\mathfrak{U}=\left\{U_{i}, I\right\}$ be given. A system $\left\{f_{i j}, i, j \varepsilon I\right\}$ of elements $f_{i j} \varepsilon E$ is called a $\mathfrak{U}$-system of transition functions if: (1) $f_{i j} \varepsilon Q_{x}-\mathfrak{m}_{x}$ for all $X \varepsilon U_{i} \cap U_{i}$ and $i, j \varepsilon I ;(2) f_{i j} f_{i h}=f_{i h}$ for all $i, j, h \varepsilon I . A$ system $\left\{f_{i}, i \varepsilon I\right\}$ of elements $f_{i} \varepsilon E$ is called a $\mathfrak{U}$-system of local generators if: (1) $f_{i} \neq 0$ for all $i \varepsilon I$; (2) $f_{i} / f_{i} \varepsilon Q_{x}-\mathfrak{m}_{x}$ for all $x \in U_{i} \cap U_{j}$ and $i, j \varepsilon I$.

Since a unique section $b(i, j) \varepsilon S\left(U_{i} \cap U_{i}, L_{0}\right)$ is associated with each $f_{i j}$, a $\mathfrak{U}$-system of transition functions is nothing but a 1 -cocycle of $\mathfrak{U}$ with values in $L_{0}$. The term "transition function" was taken from [5], section 1. To each $\mathfrak{U}$-system of local generators $\left\{f_{i}, i \varepsilon I\right\}$ is associated the $\mathfrak{U}$-system of transition functions $\left\{f_{i j}=f_{i} / f_{i}, i, j \varepsilon I\right\}$. If $f$ is the line class of $X$, represented by $\left\{f_{i} / f_{i}, i, j \varepsilon I\right\}$, we say that $\left\{f_{i}, i \varepsilon I\right\}$ is a system of local generators of $f$. Clearly, $\left\{f_{i}, i \varepsilon I\right\}$ may be regarded as a nonzero section over $X$ of the algebraic sheaf $f\left(E^{\prime}\right)$. Conversely, if $f$ is a given line class of $X$, we can always construct 
a system of local generators of $f$ as follows. Select some $\mathfrak{U}$ which admits a 1-cocycle $b$ with values in $L_{0}$ which represents $f$, and select a nonzero section $s \varepsilon S\left(X, f\left(E^{\prime}\right)\right)$. Then $s$ and $b$ together give rise as usual to a system of sections of $E^{\prime}$ over the various $U_{i}$ of $\mathfrak{U}=\left\{U_{i}, I\right\}$. This system may be regarded as a system of local generators of $f$. We observe in this connection that $f\left(E^{\prime}\right)$ has nonzero sections over $X$ galore. Namely, $f\left(E^{\prime}\right)$, considered as an algebraic sheaf, is locally isomorphic to $E^{\prime}$ whence, since $X$ is irreducible, $f\left(E^{\prime}\right)$ and $E^{\prime}$ are isomorphic $L$-sheaves. (See [1], section 36, lemma 2.) It follows that the nonzero sections of $f\left(E^{\prime}\right)$ over $X$ are in $(1,1)$-correspondence with the nonzero elements of $E$. Our notion of "local generator" corresponds to that of "Ortsfunktion" in section 15.2 of [4].

We are now going to derive a useful criterion for the cohomological equivalence of systems of local generators. Let $\mathfrak{U}=\left\{U_{i}, i \varepsilon I\right\}$ and $\mathfrak{B}=\left\{V_{i}, j \varepsilon J\right\}$ be two indexed, open coverings of $X$. Let $\left\{f_{i}, i \varepsilon I\right\}$ be a $\mathfrak{U}$-system of local generators of a line class $f$ of $X$ and let $\left\{g_{j}, j \varepsilon J\right\}$ be a $\mathfrak{B}$-system of local generators of a line class $g$ of $X$.

Criterion 4.1. $f=g$ if and only if there exists a nonzero $e \varepsilon E$ with the property that $f_{i} / g_{j} \varepsilon Q_{x}-\mathfrak{m}_{x}$ for all $i \varepsilon I, j \varepsilon J$ and $x \varepsilon U_{i} \cap V_{j}$.

Proof. Let $\mathfrak{W}=\left\{W_{a}, a \varepsilon A\right\}$ be an indexed, open covering of $X$ which is a refinement of $\mathfrak{U}$. Let the mapping $t: A \rightarrow I$ be such that $W_{a} \subset U_{t(a)}$ for all $a \varepsilon A$. Consider the $\mathfrak{M}$-system of local generators $\left\{h_{a}, a \varepsilon A\right\}$ which is defined by $h_{a}=f_{t(a)}$ for all $\alpha \varepsilon A$. The 1-cocycle of $\mathfrak{W}$ with values of $L_{0}$ which is determined by the system $\left\{h_{a b}=h_{a} / h_{b}, a, b \varepsilon A\right\}$ is the image under $t$ of the 1-cocycle of $\mathfrak{U}$ with values in $L_{0}$ which is determed by by the system $\left\{f_{i j}=f_{i} / f_{i}, i, j \varepsilon I\right\}$. Consequently, $\left\{h_{a}, a \varepsilon A\right\}$ is a system of local generators of $f$. If $\mathfrak{W}$ is a common refinement of $\mathfrak{U}$ and $\mathfrak{B}$, we denote by $z: A \rightarrow J$ and $\left\{m_{a}, a \varepsilon A\right\}$ and $\left\{m_{a b}=\right.$ $\left.m_{a} / m_{b}, a, b \varepsilon A\right\}$ the mapping and $\mathfrak{W}$-systems which arise in similar fashion from $\left\{g_{j}, j \varepsilon J\right\}$. Hence $\left\{m_{a}, a \varepsilon A\right\}$ is a system of local generators of $g$. We now proceed in two steps.

(a) There exists an e as described in Criterion 4.1. Select for $\mathfrak{W}$ any common refinement of $\mathfrak{U}$ and $\mathfrak{B}$ and put $c_{a}=e h_{a} / m_{a}$ for all $a \varepsilon A$. If $x \varepsilon W_{a}$, then $x \varepsilon U_{t(a)}$ $\cap V_{z(a)}$, whence $c_{a}=e f_{t(a)} / g_{z(a)} \varepsilon Q_{x}-\mathfrak{m}_{x}$. This shows that the system $\left\{c_{a}, a \varepsilon A\right\}$ determines a 0 -cochain of $\mathfrak{W}$ with values in $L_{0}$. The coboundary of this cochain is the 1-cocycle which is determined by the system $\left\{c_{a b}=c_{b} / c_{a}, a, b \varepsilon A\right\}$. Since $c_{b} / c_{a}=m_{a b} / h_{a b}$ for all $a, b \varepsilon A$, this proves that the 1-cocycles $\left\{h_{a b}, a, b \varepsilon A\right\}$ and $\left\{m_{a b}, a, b \varepsilon A\right\}$ are cohomologous, whence $f=g$.

(b) $f=g$. We now select the common refinement $\mathfrak{B}$ of $\mathfrak{U}$ and $\mathfrak{B}$ in such a way that the two 1-cocycles $\left\{h_{a b}, a, b \varepsilon A\right\}$ and $\left\{m_{a b}, a, b \varepsilon A\right\}$ of $\mathfrak{W}$ with values in $L_{0}$ are cohomologous; i.e., there exists a 0 -cochain $\left\{c_{a}, a \varepsilon A\right\}$ of $\mathfrak{W}$ with values in $L_{0}$ whose coboundary is the 1-cocycle $\left\{m_{a b} / h_{a b}, a, b \varepsilon A\right\}$. This means that $c_{a} \varepsilon Q_{x}-\mathfrak{m}_{x}$ for all $a \varepsilon A$ and $x \varepsilon W_{a}$ and, furthermore, that $c_{b} / c_{a}=m_{a b} / h_{a b}$ for every pair $a, b \varepsilon A$. We conclude that $c_{a} m_{a} / h_{a}$ is independent of the index $a$, 
say $c_{a} m_{a} / h_{a}=e \varepsilon E$. Select now some $i \varepsilon I, j \varepsilon J$ and $x \varepsilon U_{i} \cap V_{i}$. We finish the proof of the criterion by showing that $e f_{i} / g_{i} \varepsilon Q_{x}-\mathfrak{m}_{x}$. Accordingly, we choose an $a \varepsilon A$ in such a way that $x \varepsilon W_{a}$, whence $x \varepsilon U_{t(a)} \cap V_{z(a)}$. We infer from $x \varepsilon W_{a}$ that $c_{a} \varepsilon Q_{x}-\mathfrak{m}_{x}$, and from $x \varepsilon U_{i} \cap U_{l(a)}$ that $f_{i} / f_{t(a)} \varepsilon Q_{x}-\mathfrak{m}_{x}$, and from $x \varepsilon V_{i} \cap V_{z(a)}$ that $g_{z(a)} / g_{i} \varepsilon Q_{x}-\mathfrak{m}_{x}$. We see by direct computation that $e f_{i} / g_{j}=c_{a}\left(f_{i} / f_{t(a)}\right)\left(g_{z(a)} / g_{i}\right)$, and we are done.

Definition 4.2. A line class $f$ of $X$ is called regular if $f(L)$ has a nonzero section over $X$.

Let $f$ be a regular line class of $X$. Select some $\mathfrak{U}=\left\{U_{i}, I\right\}$ which admits a 1-cocycle $b$ with values in $L_{0}$ which represents $f$, and select a nonzero section $s \varepsilon S(X, f(L))$. This $s$ and $b$ together give rise, as before, to a $\mathfrak{U}$-system $\left\{f_{i}, i \varepsilon I\right\}$ of local generators of $f$. This system has the following special property: $f_{i} \varepsilon Q_{x}$ for all $x \in U_{i}$ and $i \varepsilon I$; we call such a system regular because each $f_{i}$ is regular over $U_{i}$ in the sense of [1], section 35. Conversely, if $f$ is any line class of $X$ which possesses a regular system of local generators, then $f(L)$ has the nonzero section over $X$ which corresponds to this system. Hence a line class $f$ of $X$ is regular if and only if $f$ possesses a regular system of local generators.

5. Line classes as divisor classes. We assume now that $X$ is a complete irreducible algebraic variety $X$. All symbols have the same meaning as in the previous section. We denote the dimension of $X$ by r, i.e. $r$ is the degree of transcendency of $E / k$. Whenever we say " $(\mathfrak{D}, \mathfrak{P})$, or just $\mathfrak{D}$ or $\mathfrak{P}$, is a prime divisor of $X$," we mean: (1) $\mathfrak{D}$ is a valuation ring of $E$ which contains $k$, and $\mathfrak{P}$ is the maximal ideal of $\mathfrak{D}$; $(2)$ if the local ring $Q$ with maximal ideal $\mathfrak{m}$ is the center of $\mathfrak{D}$ on $X$, the degree of transcendency of the field $Q / \mathrm{m}$ over $k$ is $r-1$. As usual, an integral, linear combination of prime divisors of $X$ which has at most a finite number of nonzero coefficients is called a divisor of $X$. The terms positive divisor, negative divisor, nonnegative divisor, etc. have their usual meaning. We will, furthermore, use the following terminology and notation.

(1) If $D$ is a divisor of $X$ and $\mathfrak{B}$ is a prime divisor of $X$, then $v_{\mathfrak{B}}(D)$ denotes the coefficient with which $\mathfrak{P}$ occurs in $D$. In other words, $D=\sum v_{\mathfrak{R}}(D) \mathfrak{P}$, where the sum ranges over all the prime divisors of $X$.

(2) The variety $V$ of a prime divisor $\mathfrak{D}$ of $X$ consists of all $x \varepsilon X$ with the property that $Q_{x} \subset \mathfrak{D}$. We will use that $V$ is a closed, irreducible subvariety of $X$. The variety $V(D)$ of an arbitrary divisor $D$ of $X$ is the union of the varieties of the prime divisors of $X$ which occur in $D$ with nonzero coefficients.

(3) If $x \varepsilon X$, a prime divisor $\mathfrak{D}$ of $X$ is called a local prime divisor at $x$ if $x$ belongs to the variety of that divisor; i.e. if $Q_{x} \subset \mathfrak{D}$. If $x \varepsilon X$ and $D$ is any divisor of $X$, then $D$ is called a local divisor at $x$ if $v_{\mathfrak{B}}(D) \neq 0$ implies that $\mathfrak{B}$ is a local prime divisor at $x$. If $x \varepsilon X$ and $D$ is any divisor of $X$, the local divisor $D_{x}$ of $D$ at $x$ is defined by $v_{\mathfrak{B}}\left(D_{x}\right)=0$ if $\mathfrak{P}$ is not a local prime divisor at $x$, while $v_{\mathfrak{P}}\left(D_{x}\right)=v_{\mathfrak{B}}(D)$ if $\mathfrak{P}$ is a local prime divisor at $x$. In particular, if $e$ is a nonzero 
element of $E$, not only its divisor (e), but also its local divisor $(e)_{x}$ is well defined for all $x \varepsilon X$.

(4) The symbol $\equiv$ is used to denote linear equivalence of divisors. Precisely, if $D_{1}$ and $D_{2}$ are divisors of $X$, then $D_{1} \equiv D_{2}$ if and only if $D_{1}-D_{2}=(e)$ for some nonzero $e \varepsilon E$.

We need the following proposition.

Proposition 5.1. Let $\mathfrak{U}=\left\{U_{i}, i \varepsilon I\right\}$ be given. Suppose that $\left\{D_{i}, i \varepsilon I\right\}$ is a system of divisors of $X$ which has the following property: $\left(D_{i}\right)_{x}=\left(D_{i}\right)_{x}$ for all $x \varepsilon U_{i} \cap U_{i}$ and $i, j \varepsilon I$. Then there exists a unique divisor $D$ of $X$ with the property that $D_{x}=\left(D_{i}\right)_{x}$ for all $x \varepsilon U_{i}$ and $i \varepsilon I$.

Proof. It is obvious that there can exist at most one divisor of $X$ with the required property. In order to show that there exists one, we proceed in two steps.

(a) Let $(\mathfrak{D}, \mathfrak{B})$ be a prime divisor of $X$, let $x$ and $y$ be two points on the variety $V$ of $\mathfrak{D}$, and let $x \in U_{i}$ and $y \varepsilon U_{i}$ for $i, j \varepsilon I$. We assert that then $v_{\mathfrak{B}}\left(\left(D_{i}\right)_{x}\right)=$ $v_{\mathfrak{B}}\left(\left(D_{i}\right)_{y}\right)$. Namely, since $V \cap U_{i}$ and $V \cap U_{i}$ are two open, nonempty subsets of the irreducible topological space $V$, there exists a point $z \varepsilon V \cap U_{i} \cap U_{i}$. (See [1], section 30, for irreducible spaces.) We conclude that $\left(D_{i}\right)_{z}=\left(D_{i}\right)_{z}$ which implies, since $z \varepsilon V$, that $v_{\mathfrak{B}}\left(D_{i}\right)=v_{\mathfrak{B}}\left(D_{i}\right)$; the assertion follows trivially from this last equality. We denote by $v_{\mathfrak{B}}$ this unique integer $v_{\mathfrak{B}}\left(\left(D_{\boldsymbol{i}}\right)_{x}\right)$ which the system $\left\{D_{i}, i \varepsilon I\right\}$ associates with $\mathfrak{P}$.

(b) $v_{\mathfrak{B}}=0$, except for at most a finite number of prime divisors $\mathfrak{B}$ of $X$. Namely, since $X$ is compact (we do not include the Hausdorff axiom in the definition of compactness), there exist a finite number of indices $i_{1}, \cdots, i_{n} \varepsilon I$ such that $X=U_{i_{1}} \cup \ldots \cup U_{i_{n}}$. Clearly, if $v_{\mathfrak{B}} \neq 0$, then $\mathfrak{P}$ occurs with nonzero coefficient in one of the divisors $D_{i_{1}}, \cdots, D_{i_{n}}$, which proves (b). The divisor $D=\sum v_{\mathfrak{B}} \mathfrak{B}$, where the sum ranges over all the prime divisors of $X$, clearly satisfies the requirement of Proposition 5.1.

Let $f$ be a line class of $X$ and $\left\{f_{i}, i \varepsilon I\right\}$ a $\mathfrak{U}$-system of local generators of $f$, where $\mathfrak{U}=\left\{U_{i}, i \varepsilon I\right\}$. The system of divisors $\left\{\left(f_{i}\right), i \varepsilon I\right\}$ possesses the property stated in Proposition 5.1; namely, if $i, j \varepsilon I$ and $x \varepsilon U_{i} \cap U_{i}$, then $\left(f_{i}\right)_{x}=$ $\left(f_{i} / f_{j}\right)_{x}+\left(f_{i}\right)_{x}=\left(f_{i}\right)_{x}$ because $f_{i} / f_{j} \varepsilon Q_{x}-\mathfrak{m}_{x}$. Consequently, we can associate a unique divisor $D_{1}$ of $X$ with the system $\left\{f_{i}, i \varepsilon I\right\}$. Consider now a second line class $g$ of $X$ and a $\mathfrak{B}$-system $\left\{g_{j}, j \varepsilon J\right\}$ of local generators of $g$, where $\mathfrak{B}=$ $\left\{V_{i}, j \varepsilon J\right\}$ is also an indexed, open covering of $X$. Let $D_{2}$ be the divisor of $X$ associated with $\left\{g_{j}, j \varepsilon J\right\}$.

Proposition 5.2. If $f=g$, then $D_{1} \equiv D_{2}$. If $D_{1} \equiv D_{2}$ and $X$ is normal, $f=g$.

Proof. Let $f=g$ and let $e \varepsilon E$ be as described in Criterion 4.1. Select $x \varepsilon X$, and then $i \varepsilon I$ and $j \varepsilon J$ in such a way that $x \in U_{i} \cap V_{j}$. We conclude from $e f_{i} / g_{i} \varepsilon Q_{x}-\mathfrak{m}_{x}$ that $\left(D_{2}\right)_{x}-\left(D_{1}\right)_{x}=(e)_{x}$, whence $D_{1} \equiv D_{2}$. Conversely, let 
$D_{1} \equiv D_{2}$ and let $X$ be normal. Select $e \varepsilon E$ such that $D_{2}-D_{1}=(e)$. Then $\left(e f_{i} / g_{j}\right)_{x}=0$ for all $i \varepsilon I$ and $j_{\varepsilon} J$ and $x \varepsilon U_{i} \cap V_{j}$. Since $X$ is normal, it follows that $e f_{i} / g_{i} \varepsilon Q_{x}-\mathfrak{m}_{x}$, and Criterion 4.1 now tells us that $f=g$.

A divisor $D$ of $X$ is called locally linearly equivalent to zero if there exists a $\mathfrak{U}=\left\{U_{i}, i \varepsilon I\right\}$ and a system $\left\{e_{i}, i \varepsilon I\right\}$, where $e_{i} \varepsilon E$ and $e_{i} \neq 0$, such that $D_{x}=\left(e_{i}\right)_{x}$ for all $x \varepsilon U_{i}$ and $i \varepsilon I$. We observe that we cannot conclude that $\left\{e_{i}, i \varepsilon I\right\}$ is a $\mathfrak{U}$-system of local generators unless $X$ is normal. It is clear that if $D_{1} \equiv D_{2}$, then both these divisors are locally linearly equivalent to zero as soon as one of them is. In case $X$ is nonsingular or, more generally, if each local ring of $X$ is a unique factorization domain, all divisors of $X$ are evidently locally linearly equivalent to zero. In general, however, even if $X$ is normal, $X$ will have divisors which are not locally linearly equivalent to zero; see, for instance, example 10.2 .

If $f$ is a line class of $X$, we denote by $\|f\|$ the unique divisor class (with respect to linear equivalence) of $X$ which, according to Proposition 5.2, is associated with $f$. We obtain all the divisors of $\|f\|$ from the various systems of local generators of $f$, whence they are locally linearly equivalent to zero. If $X$ is normal, we obtain in this way all the divisor classes of $X$ whose divisors are locally linearly equivalent to zero. (If $X$ is not normal, the divisor classes whose divisors are locally linearly equivalent to zero correspond to the fibre classes, in the sense of Definition 1.1, of the sheaf of rings $L^{*}$. The stalk $\left(L^{*}\right)_{x}$ is the integral closure of $L_{x}$ in $E_{x}^{\prime}$; see [6] for the sheaf $L^{*}$.) The mapping $f \rightarrow\|f\|$ is clearly a group homomorphism from the multiplicative group $H^{1}\left(X, L_{0}\right)$ into the additive group of the divisor classes of $X$ whose divisors are locally linearly equivalent to zero. We see from Proposition 5.2 and the above remarks that this homomorphism is an isomorphism if $X$ is normal. We denote by $|f|$ the set of the nonnegative divisors which belong to $\|f\|$; of course, $|f|$ may be empty. We have fixed our notation in such a way that if $D \varepsilon\|f\|$, then $|D|=|f|$, where $|D|$ denotes, as customary, the complete linear system which is associated with $D$.

We will use the following notation: If $R$ is a subring of $E$, then $R^{*}$ is the integral closure of $R$ in $E$. In particular, $k^{*}$ is the algebraic closure of $k$ in $E$.

Proposition 5.3. If $f$ is regular, $|f| \neq \varnothing$. In that case, there exists a natural mapping $\lambda$ from the set of nonzero sections of $f(L)$ over $X$ into $|f|$; two nonzero sections of $f(L)$ over $X$ give rise to the same divisor of $|f|$ if and only if they differ by an element of $k^{*}$. If $X$ is normal, $\lambda$ is onto. Finally, if $|f| \neq \varnothing$ and $X$ is normal, $f$ is regular.

Proof. Let $f$ be regular and let $b$ be a 1-cocycle of $\mathfrak{U}=\left\{U_{i}, i \varepsilon I\right\}$ with values in $L_{0}$ which represents $f$. Let $\left\{f(L), u_{i}\right\}$ be obtained by means of $L$ and $b$; as always, $u_{i}:(f(L))\left(U_{i}\right) \rightarrow L\left(U_{i}\right)$ is an $L\left(U_{i}\right)$-isomorphism for each $i \varepsilon I$, and the usual conditions are satisfied. A nonzero section $s$ of $f(L)$ over $X$, together with $b$, gives rise to a regular $\mathfrak{U}$-system $\left\{f_{i}, i \varepsilon I\right\}$ of local generators of $f$ (see section 4). Clearly, the divisor of $X$ associated with $\left\{f_{i}, i \varepsilon I\right\}$ is nonnegative and we denote this divisor by $(s)$. The mapping $s \rightarrow(s)$ is the mapping $\lambda$ referred 
to in Proposition 5.3. If $s^{\prime}$ is another nonzero section of $f(L)$ over $X$, we show easily that the regular $\mathfrak{U}$-system of local generators of $f$ which arises from $s^{\prime}$ and $b$ is $\left\{f_{i} e, i \varepsilon I\right\}$, for some nonzero $e \varepsilon E$. Obviously, $\left(s^{\prime}\right)=(s)+(e)$, whence $\left(s^{\prime}\right)=(s)$ if and only if $e \varepsilon k^{*}$. By letting $s^{\prime}$ vary and keeping $s$ fixed, we obtain all those nonzero $e \varepsilon E$ which have the property that $f_{i} e \varepsilon Q_{x}$ for all $x \varepsilon U_{i}$ and $i \varepsilon I$. On the other hand, $(s)+(e) \geqq 0$ if and only if $f_{i} e \varepsilon Q_{x}^{*}$ for all $x \varepsilon U_{i}$ and $i \varepsilon I$. Hence if $X$ is normal, $\lambda$ is onto. In order to prove the last sentence of Proposition 5.3, let $X$ be normal and $|f| \neq \varnothing$. Then there exists a $\mathfrak{U}=\left\{U_{i}, i \varepsilon I\right\}$ and a $\mathfrak{U}$-system of local generators $\left\{f_{i}, i \varepsilon I\right\}$ of $f$ with the property that $\left(f_{i}\right)_{x} \geqq 0$ for all $x \varepsilon U_{i}$ and $i \varepsilon I$. Since $X$ is normal, this means precisely that $f_{i} \varepsilon Q_{x}$ for all $x \varepsilon U_{i}$ and $i \varepsilon I$, i.e. that $\left\{f_{i}, i \varepsilon I\right\}$ and hence $f$ is regular.

Let $s, s^{\prime}$ and $\left\{f_{i} e, i \varepsilon I\right\}$ be as in the proof of Proposition 5.3. By keeping $s$ fixed and letting $s^{\prime}$ vary, we obtain a mapping $s^{\prime} \rightarrow e$ from the nonzero elements of $H^{0}(X, f(L))$ into $E$. If we map the zero element of $H^{0}(X, f(L))$ on the zero element of $E$, this mapping becomes a $k$-linear isomorphism from $H^{0}(X, f(L))$ onto some $k$-linear subspace $N$ of $E$. We see from Proposition 5.3 that if $X$ is normal, the set of divisors $(s)+(e)$, where $e$ runs through the nonzero elements of $N$, is precisely $|f|$. Hence if $X$ is normal and $k=k^{*}$, the classical dimension of the complete linear system $|f|$ is $\operatorname{dim}_{k}(N)-1$. (The condition $k=k^{*}$ is imposed only in order that this classical dimension will be well defined.) Following the custom of denoting the $k$-dimension of a cohomology group $H^{t}$ by $h^{t}$, we have found

Proposition 5.4. If $k=k^{*}$ and $X$ is normal, $\operatorname{dim}(|f|)=h^{0}(X, f(L))-1$.

6. The mapping $w: F \rightarrow f\left(F^{\prime}\right)$ for regular $f$. We continue the notations of the proof of Proposition 5.3. Hence $X$ is a complete irreducible algebraic variety, $f$ is a regular line class of $X$, and $b, \mathfrak{U}=\left\{U_{i}, i \varepsilon I\right\},\left\{f(L), u_{i}\right\}, s,(s)$ and $\left\{f_{i}, i \varepsilon I\right\}$ are all as stated in that proof. We repeat that $\left\{f_{i}, i \varepsilon I\right\}$ arises from $s$ and $b$, whence $f_{i} \varepsilon Q_{x}$ for all $x \varepsilon U_{i}$ and $i \varepsilon I$. We denote by $V$ the variety of the nonnegative divisor $(s)$; see section 5 for the notion of the variety of a divisor. For each $x \in X$, the $L_{x}$-module $(f(L))_{x}$ is $L_{x}$-isomorphic with $L_{x}$, whence $(f(L))_{x}$ possesses a unique maximal $L_{x}$-submodule which we denote by $\mathfrak{n}_{x}$.

Proposition 6.1. $V$ consists of those points $x \in X$ which have the property that $s(x) \varepsilon \mathfrak{n}_{x}$; i.e., if $i \varepsilon I$ and $x \varepsilon U_{i}$, then $x \varepsilon V \cap U_{i}$ if and only if $f_{i} \varepsilon \mathfrak{m}_{x}$. It follows that if $X$ is normal and $x \varepsilon X$, then $x$ is a base point of $|f|$ if and only if $s^{\prime}(x) \varepsilon \mathfrak{n}_{x}$ for all $s^{\prime} \varepsilon S(X, f(L))$.

Proof. Let $i \varepsilon I$ and $x \varepsilon U_{i}$. Obviously, $x \varepsilon V$ if and only if $(s)_{x}>0$, i.e. if and only if $\left(f_{i}\right)_{x}>0$. This is equivalent to saying that $f_{i} \varepsilon \mathfrak{m}_{x}$, i.e. that $s(x) \varepsilon \mathfrak{n}_{x} \cdot$ Now let $X$ be normal. We know from Proposition 5.3 that $|f|$ then consists of the divisors $\left(s^{\prime}\right)$, where $s^{\prime}$ runs through the nonzero sections of $f(L)$ over $X$. By the definition of base point, the point $x \varepsilon X$ is a base point of $|f|$ if and only if the varieties of all the divisors $\left(s^{\prime}\right)$ pass through $x$, i.e. if and only if $s^{\prime}(x) \varepsilon \mathfrak{n}_{x}$ 
for all nonzero $s^{\prime} \varepsilon S(X, f(L))$. If $\omega$ is the zero section of $f(L)$ over $X$, then, of course, $\omega(x)=0 \varepsilon \mathfrak{n}_{x}$.

We now consider an algebraic coherent sheaf $F$ of $X$ whose torsion sheaf we denote by $T$; see [2], section 2 , for torsion sheaves. We know from section 3 (of the present paper) that $s$ gives rise to an $L$-homomorphism $w: F \rightarrow f(F)$.

Proposition 6.2. Sup (coker $(w))=V \cap \operatorname{Sup}(F)$ and Sup $(\operatorname{ker}(w)) \subset V \cap$ $\operatorname{Sup}(T)$.

Proof. Let $s_{i}$ be the section of $L$ over $U_{i}$ which is determined by $f_{i}$. If $x \varepsilon U_{i}$, then $x \varepsilon$ Sup (coker $(w)$ ) if and only if $s_{i}(x) F_{x} \neq F_{x}$, according to Proposition 3.2. We conclude from [9], Chapter VIII, Proposition 5.1', that this is equivalent to saying that $F_{x} \neq 0$ and $s_{i}(x)$ belongs to the unique maximal ideal $\mathfrak{p}_{x}$ of $L_{x}$, i.e. that $x \varepsilon \operatorname{Sup}(F)$ and $f_{i} \varepsilon \mathfrak{m}_{x}$. It now follows from Proposition 6.1 that Sup $($ coker $(w))=V \cap \operatorname{Sup}(F)$. If $x \varepsilon U_{i} \cap \operatorname{Sup}($ ker $(w))$, there exists a nonzero $g \varepsilon F_{x}$ such that $s_{i}(x) g=0$, according to Proposition 3.2. This obviously implies that $s_{i}(x) \varepsilon \mathfrak{p}_{x}$ and $x \varepsilon \operatorname{Sup}(T)$, i.e. that $x \varepsilon V \cap \operatorname{Sup}(T)$.

We will see in section 8 that $\operatorname{Sup}(\operatorname{ker}(w))$ may be different from $V \cap \operatorname{Sup}(T)$.

Consider the sheaf $B$ of principal ideals of $X$ which is defined by $B_{x}=s_{i}(x) L_{x}$ for all $x \in U_{i}$ and $i \varepsilon I$. We have seen at the end of section 3 that $B=\operatorname{Im}\left(w^{\prime}\right)$, where $w^{\prime}: f^{-1}(L) \rightarrow L$ is the $L$-homomorphism which arises from $s$ and $f^{-1}(L)$. It follows that $B$ is a coherent sheaf of ideals of $X$.

Proposition 6.3. $B \simeq f^{-1}(L)$ and $\operatorname{Var}(B)=V$.

Proof. Since $f^{-1}(L)$ is locally isomorphic with $L$ and since $L$ has zero torsion, the same is true for $f^{-1}(L)$. Hence, according to Proposition 6.2, Sup $\left(\operatorname{ker}\left(w^{\prime}\right)\right)=\varnothing$, i.e. $w^{\prime}$ is a monomorphism. This proves that $B \simeq f^{-1}(L)$. Furthermore, $x \varepsilon U_{i} \cap \operatorname{Var}(B)$ if and only if $s_{i}(x) L_{x} \neq L_{x}$, i.e. if and only if $f_{i} \varepsilon \mathfrak{m}_{x}$. We now conclude from Proposition 6.1 that $\operatorname{Var}(B)=V$.

We should be careful to observe that the model of $f^{-1}(L)$ which $B$ provides depends not only on the choice of $f$ and $b$, but also on the choice of $s$. This is brought out further by the observation that in the system $\left\{B=f^{-1}(L), u_{i}^{\prime}\right\}$ the $L\left(U_{i}\right)$-isomorphism $u_{i}^{\prime}: B\left(U_{i}\right) \rightarrow L\left(U_{i}\right)$ is defined by $u_{i}^{\prime}(g)=a$ if $x \varepsilon U_{i}$ and $g=s_{i}(x) a$ for some $a \varepsilon L_{x}$.

Let $X$ be normal. If $x \varepsilon U_{i}$ then $(s)_{x}=\left(f_{i}\right)_{x}$, whence $(s)_{x}$ is without multiple components if and only if the ideal $f_{i} Q_{x}$ of the integrally closed ring $Q_{x}$ is equal to its radical. Hence $(s)$ is without multiple components if and only if, for each $i \varepsilon I$ and $x \varepsilon U_{i}$, the ideal $s_{i}(x) L_{x}$ of $L_{x}$ is equal to its radical. In order not to have to mention over and over that the zero divisor is an exception, we consider the zero divisor as a divisor without multiple components. Of course, $(s)=$ $0 \leftrightarrow B=L \leftrightarrow V=\varnothing$, and in this case "the sheaf of local rings of $V$ " must be considered as the zero sheaf. We now return to the study of $w$ and $B$.

Proposition 6.4. Let $X$ be normal. Then $(s)$ is without multiple components if and only if the quotient sheaf $L / B$ (restricted to $V$ ) is algebraic coherent over $V$. In 
this case, $L / B$ may be regarded as the sheaf of local rings of $V$ and coker $(w)$ (restricted to $V$ ) is algebraic coherent over $V$.

Proof. We have just seen that (s) is without multiple components if and only if $B_{x}=$ radical $\left(B_{x}\right)$ for all $x \varepsilon X$. Proposition 6.4 is hence a corollary of Proposition 4.1 , except for the statement concerning coker $(w)$. We know from Proposition 6.2 that coker $(w)$ is zero outside $V$. Consequently, according to section 39 of [1], coker $(w)$ (restricted to $V$ ) is algebraic coherent over $V$ if and only if the annihilator $\mathfrak{a}$ of coker $(w)$ contains the annihilator $\mathfrak{b}$ of the sheaf of local rings (extended by zero over $X$ ) of $V$. Proposition 6.3 tells us that $V=\operatorname{Var}(B)$, whence we conclude from Proposition 4.1 that $\mathfrak{b}_{x}$ is the radical of $B_{x}=s_{i}(x) L_{x}$ for all $x \in U_{i}$. It is obvious that $\mathfrak{a}_{x} \supset s_{i}(x) L_{x}$, because of the fact that (coker $(w))_{x}=(f(F))_{x} / s_{i}(x)\left(f\left(F^{\prime}\right)\right)_{x}$. We remarked above that if $X$ is normal and $(s)$ is without multiple components, $s_{i}(x) L_{x}$ is equal to its radical.

If we use the algebraic coherent sheaf $f^{-1}(F)$ of $X$ in place of $F$, we obtain an $L$-homomorphism $w^{*}$ from $f^{-1}(F)$ into $f\left(f^{-1}(F)\right) \simeq F$. All our results apply, of course, to this $w^{*}: f^{-1}(F) \rightarrow F$. Since $f^{-1}(F)$ and $F$ are locally $L$-isomorphic, they have the same supports and their torsion sheaves also have the same supports. Hence if we apply Proposition 6.2 to $w^{*}$, we find that Sup (coker $\left.\left(w^{*}\right)\right)=V \cap \operatorname{Sup}(F)$ and that $\operatorname{Sup}\left(\operatorname{ker}\left(w^{*}\right)\right) \subset V \cap \operatorname{Sup}(T)$. The isomorphism $B \simeq f^{-1}(L)$ gives rise to an exact sequence $0 \rightarrow f^{-1}(L) \rightarrow L \rightarrow L / f^{-1}(L) \rightarrow 0$, where $f^{-1}(L) \rightarrow L$ is the inclusion mapping $\mu$ of $B$ into $L$. If we tensor this sequence with $F$, we obtain the exact sequence $0 \rightarrow \operatorname{ker}(c) \rightarrow f^{-1}(L) \otimes_{L} F \rightarrow$ $L \otimes_{L} F \rightarrow L / f^{-1}(L) \otimes_{L} F \rightarrow 0$, where $c: f^{-1}(L) \otimes_{L} F \rightarrow L \otimes_{L} F$ is the tensor product of $\mu$ with the identity mapping of $F$ onto itself. We know that $f^{-1}(L) \otimes_{L} F \simeq f^{-1}(F)$ and that $L \otimes_{L} F \simeq F$, and we prove easily that there exists a commutative diagram

$$
\begin{array}{ccc}
f^{-1}(L) \otimes_{L} F & \stackrel{c}{\rightarrow} L \otimes_{L} F \\
\simeq \downarrow & & \downarrow \simeq \\
f^{-1}(F) & \underset{w *}{\rightarrow} & F
\end{array}
$$

Since the two vertical arrows are isomorphisms, $\operatorname{ker}(c) \simeq \operatorname{ker}\left(w^{*}\right)$ and $L / f^{-1}(L) \otimes_{L} F \simeq$ coker $\left(w^{*}\right)$. This "tensor approach" was used in [1], section 81 , in the special case that $X$ is projective and $f$ is the line class arising from a hyperplane section of $X$.

7. Concrete models for $f(F)$. We return to the case that $f$ is an arbitrary line class of our irreducible, complete $X$, where $f$ is not necessarily regular. We assume that $\left\{f_{i}, i \varepsilon I\right\}$ is a $\mathfrak{U}$-system of local generators of $f$, where $\mathfrak{U}=\left\{U_{i}, I\right\}$. The sheaf $W$ of fractional ideals of $X$ whose stalk $x \varepsilon U_{i}$ is the fractional ideal $\left(1 / f_{i}\right) Q_{x}$ may always be used as a model of $f(L)$. (See [1], section 40 , or [6] for sheaves of fractional ideals.) Namely, consider the system $\left\{W, u_{i}\right\}$, where the $L\left(U_{i}\right)$-isomorphism $u_{i}: W\left(U_{i}\right) \rightarrow L\left(U_{i}\right)$ is defined by $u_{i}(g)=f_{i} g$ if $i \varepsilon I$ and $x \varepsilon U_{i}$ and $g \varepsilon\left(1 / f_{i}\right) Q_{x}$. We see immediately that $u_{i}=\left(f_{i} / f_{i}\right) u_{j}$ over $U_{i} \cap U_{i}$, 
whence $W$ is indeed a good, concrete model of $f(L)$. We conclude from Proposition 2.2 that if $F$ is an algebraic sheaf of $X$, then $W \otimes_{L} F$ is a (concrete) model of $f(F)$.

Let now $D$ be the divisor of $X$ associated with the system $\left\{f_{i}, i \varepsilon I\right\}$. If $e$ is a nonzero element of $E$ and $x \varepsilon U_{i}$, then $(e)_{x}+D_{x} \geqq 0$ if and only if $e f_{i} \varepsilon Q_{x}^{*}$. Hence the customary sheaf $L(D)$, associated with $D$, is the sheaf of fractional ideals of $X$ whose stalk at $x \varepsilon U_{i}$ is the fractional ideal $\left(1 / f_{i}\right) Q_{x}^{*}$. (See, for instance, [6] for the sheaf $L(D)$.) We conclude that if $X$ is normal, the sheaves $f(L)$ and $L(D)$ may be identified.

We now assume that $f$ is regular and that the system $\left\{f_{i}, i \varepsilon I\right\}$ is, as in the previous section, a regular $\mathfrak{U}$-system of local generators of $f$. Then $Q_{x} \subset\left(1 / f_{i}\right) Q_{x}$ for all $x \varepsilon U_{i}$ and $i \varepsilon I$, whence $L \subset W$. Consequently, we have an exact sequence $0 \rightarrow L \rightarrow f(L) \rightarrow f(L) / L \rightarrow 0$, where $L \rightarrow f(L)$ is the inclusion mapping $\mu$ of $L$ into $W$. If we tensor this sequence with an algebraic sheaf $F$ of $X$, we obtain the exact sequence $0 \rightarrow \operatorname{ker}(c) \rightarrow L \otimes_{L} F \rightarrow f(L) \otimes_{L} F \rightarrow f(L) / L \otimes_{L} F \rightarrow 0$ where $c: L \otimes_{L} F \rightarrow f(L) \otimes_{L} F$ is the tensor product of $\mu$ with the identity mapping of $F$ onto itself. We know that $L \otimes_{L} F \simeq F$ and that $f(L) \otimes_{L} F \simeq f(F)$, and we prove easily that we have a commutative diagram

$$
\begin{array}{ccc}
L \otimes_{L} F & \stackrel{c}{\rightarrow} & f(L) \otimes_{L} F \\
\simeq \downarrow \downarrow & & \downarrow \simeq \widetilde{\simeq} \\
F & \vec{w} & f(F)
\end{array}
$$

Here $w$ has the same meaning as in the previous section and the vertical arrows are isomorphisms. It follows that $\operatorname{ker}(c) \simeq \operatorname{ker}(w)$ and $f(L) / L \otimes_{L} F \simeq$ coker $(w)$.

We see that the sheaf $B$ of the previous section is "the concrete model $W$ of $f^{-1}(L)$." Hence if $X$ is normal, $B$ may be identified with the sheaf $L(-D)$, where $D$ is again the divisor of the system $\left\{f_{i}, i \varepsilon I\right\}$.

8. Projective varieties. We assume everywhere in this section that our $X$ is an irreducible, projective variety. The symbols $E, E^{\prime}, L, L_{0}$ and $k$ retain their previous meanings. We review in a few lines all that we need about "projective classes of modules"; for details, see [8] or section 7 of [7].

Let $C$ be the projective class of affine coordinate modules of $X$. This means:

(1) An element $M \varepsilon C$ is a finite dimensional subspace of $E$, considered as a vector space over $k$. Furthermore, $1 \varepsilon M$.

(2) If $M_{1}$ and $M_{2}$ belong to $C$, then $M_{1}=e M_{2}$ for some nonzero $e \varepsilon E$. (Observe that this implies that $1 / e \varepsilon M_{2}$.) If $M \varepsilon C$ and $e$ is a nonzero element of $E$, also $(1 / e) M \varepsilon C$.

(3) If $M \varepsilon C$ and $\mathfrak{p}$ is a nonzero, nonunit prime ideal of $k[M]$, the ring of quotients $(k[M])_{\text {„ }}$ is a local ring of $X$. We obtain all the local rings of $X$ by letting $M$ run through $C$ and $\mathfrak{p}$ through $k[M]$. It follows that $E=k(M)$, for all $M \varepsilon C$. 
The above three properties determine $C$ within biregular equivalence. (Two projective classes of modules $C_{1}$ and $C_{2}$ of $E$ are called biregularly equivalent if they give rise to the same set of local rings, i.e., if their associated projective varieties are biregularly equivalent in the usual sense.) This finishes our review of projective classes of modules.

We now select an $M \varepsilon \subset$ and nonzero elements $a_{0}, \cdots, a_{m} \varepsilon M$ which generate the $k$-vector space $M$; it is not necessary that $a_{0}, \cdots, a_{m}$ be linearly independent over $k$. With each module $\left(1 / a_{i}\right) M$ of $E$ is associated the open subset $U_{i}$ of $X$, where $x \varepsilon U_{i}$ if and only if $\left(1 / a_{i}\right) M \subset Q_{x}$. (See section 7 of [7]. "Module of $E$ " means "finite dimensional subspace of the $k$-vector space $E . ")$ We know from that same reference that $X=U_{0} \cup \ldots \cup U_{m}$, and we denote this indexed, open covering of $X$ by $\mathfrak{u}$. If $0 \leqq i, j \leqq m$ and $x \varepsilon U_{i} \cap U_{i}$, then $\left(1 / a_{i}\right) M$ and $\left(1 / a_{i}\right) M$ belong to $Q_{x}$, whence $a_{i} / a_{i}$ and $a_{i} / a_{i}$ both belong to $Q_{x}$. This shows that $a_{i} / a_{i} \varepsilon Q_{x}-\mathfrak{m}_{x}$, from which it follows that the system $\left\{1 / a_{i}, i=0, \cdots, m\right\}$ is a $\mathfrak{U}$-system of local generators. Suppose now that we select some other $N$ \& $C$ and nonzero elements $b_{0}, \cdots, b_{n} \varepsilon N$ which generate the $k$-vector space $N$. We then obtain a second indexed, open covering $\mathfrak{B}$ of $X=V_{0} \cup \ldots \cup V_{n}$ and a $\mathfrak{B}$-system of local generators $\left\{1 / b_{i}, j=0, \cdots, n\right\}$.

Proposition 8.1. The systems $\left\{1 / a_{i}, i=0, \cdots, m\right\}$ and $\left\{1 / b_{i}, j=0, \cdots, n\right\}$ represent the same line class of $X$.

Proof. There exists a nonzero $e$ \& $M$ such that $(1 / e) M=N$. Let $0 \leqq i \leqq m$ and $0 \leqq j \leqq n$ and $x \varepsilon U_{i} \cap V_{i}$. Then, since $x \varepsilon U_{i}, e b_{i} / a_{i} \varepsilon\left(e / a_{i}\right) N=\left(1 / a_{i}\right)$ $M \subset Q_{x} ;$ and since $x \varepsilon V_{i}, a_{i} / e b_{i} \varepsilon\left(1 / e b_{i}\right) M=\left(1 / b_{i}\right) N \subset Q_{x}$. This shows that $e b_{i} / a_{i} \varepsilon Q_{x}-\mathfrak{m}_{x}$, and Proposition 8.1 now follows immediately from Criterion 4.1.

The unique line class of $X$ which is determined by the systems $\left\{1 / a_{i}, i=0\right.$, $\cdots, m\}$ of Proposition 8.1 is denoted by $p$. We know that $x \varepsilon U_{i}$ if and only if $\left(1 / a_{i}\right) M \subset Q_{x}$, which implies that $1 / a_{i} \varepsilon Q_{x}$. We conclude that $\left\{1 / a_{i}, i=\right.$ $0, \cdots, m\}$ is a regular system of local generators and, consequently, that $p$ is a regular line class of $X$. It is easy to show (but we do not need it) that, for all $n \geqq 1$, the divisor class $\left\|p^{n}\right\|$ is the class of the divisors which are "cut out on $X$ by the hypersurfaces of degree $n$ of the ambient projective space."

Let $F$ be an algebraic coherent sheaf of $X$ and let $n \varepsilon Z$. ( $Z$ denotes the ring of the rational integers.) The definition of the sheaf $F(n)$, given in section 66 of [1], shows clearly that we may identify $F(n)$ with $p^{n}(F)$.

Proposition 8.2. Let $f$ be a line class of $X$. There exists an $n_{0} \varepsilon Z$ such that, for all $n \geqq n_{0}$, the line class $p^{n} f$ is regular and the linear system $\left|p^{n} f\right|$ is without base points.

Proof. We denote the algebraic coherent sheaf $f(L)$ of $X$ by $F$. We choose $n_{0} \varepsilon Z$ in such a way that, for all $n \geqq n_{0}$ and all $x \varepsilon X$, the $L_{x}$-module $\left(p^{n}(F)\right)_{x}$ is generated by the elements of $S\left(X, p^{n}(F)\right)$; this is possible, according to Theorem 2 of section 66 of [1]. Let now $n \geqq n_{0}$ and $x \in X$ and let $\mathfrak{n}_{x}$ be the unique maximal 
$L_{x}$-submodule of $\left(p^{n} f(L)\right)_{x}$. The meaning of $n_{0}$ implies that there exists an $s \varepsilon S\left(X, p^{n} f(L)\right)$ with the property that $s(x) \notin \mathfrak{n}_{x}$. Such an $s$ is obviously nonzero, whence $p^{n} f$ is regular, according to Definition 4.2. Furthermore, we conclude from Proposition 6.1 that $x$ does not belong to the variety of the divisor $(s)$ of $X$ which is associated with $s$. Since $(s) \varepsilon\left|p^{n} f\right|$, the complete linear system $\left|p^{n} f\right|$ is without base points.

Corollary 8.1. The multiplicative group $H^{1}\left(X, L_{0}\right)$ is generated by the regular line classes.

Proof. Let $f$ be a line class of $X$. Select $n \varepsilon Z$ in such a way that $p^{n} f$ is a regular line class. Since $p$ is a regular line class and $f=\left(p^{n} f\right) p^{-n}$, we are done.

We now return to the study of Proposition 6.2 which, since $X$ is projective, can be sharpened as follows. Select a fixed module $M_{\varepsilon} C$ and an element $t$ which is transcendental over $E$. The field $E(t)$ has degree of transcendency 1 over $E$ and the graded ring $\mathfrak{D}=k[M t] \subset E(t)$ is the homogeneous coordinate ring of $C$ (or of $X$, whichever language one prefers; for the details of this construction of the homogeneous coordinate ring of a projective variety, see [6]). Let $F$ be an algebraic coherent sheaf of $X$ and $\mathfrak{M}$ a Noetherian o-module whose associated sheaf is $F$; see [1], sections $56-60$, for the construction of $\mathfrak{M}$. We denote the associated prime ideals of the zero module of $\mathfrak{M}$ by $\mathfrak{p}_{1}, \cdots, \mathfrak{p}_{n}$. Each $\mathfrak{p}_{i}$ is a prime ideal of $\mathrm{D}$, and a closed irreducible subvariety $Y_{j}$ of $X$ is associated with it. It may very well happen that $Y_{i} \subset Y_{i}$ for some $1 \leqq i \neq j \leqq n$; as a matter of fact, $X$ may occur among the $Y_{i}^{\prime}$ 's. We know from [1], section 81, that Sup $(F)=Y_{1} \cup \ldots \cup Y_{n}$, and we now derive the corresponding statement for the torsion sheaf $T$ of $F$.

Proposition 8.3. Sup $(T)$ is the union of those $Y_{j}$ 's which are distinct from $X$.

Proof. Let $x \in X$. Then $x \in$ Sup $(T)$ if and only if the zero module $\omega$ of the Noetherian $L_{x}$-module $F_{x}$ has an associated prime ideal which is a nonzero, nonunit prime ideal of $L_{x}$. If $\mathfrak{p}$ is the homogeneous prime ideal of $\mathfrak{o}$ which corresponds to $x$, then $\omega$ has a nonzero, nonunit associated prime ideal if and only if $\mathfrak{p}$ contains a $\mathfrak{p}_{j} \neq 0$ for some $1 \leqq j \leqq n$. (See section 81 of [1] for details.) Since this is equivalent to saying that $x \varepsilon Y_{i}$ for some $Y_{i} \neq X$, we are done.

Let $f$ be a regular line class of $X$ and $s$ a nonzero section of $f(L)$ over $X$. The support of the cokernel of the resulting $L$-homomorphism $w: F \rightarrow f(F)$ was described completely in Proposition 6.2. In order to describe also the support of the kernel of $w$ completely, let $V$ again denote the variety of the divisor (s) of $X$ which is associated with $s$.

Proposition 8.4. Sup $(\operatorname{ker}(w))$ is the union of those $Y_{i}$ 's which are subvarieties of $V$.

Proof. Let $\mathfrak{U}=\left\{U_{i}, i \varepsilon I\right\}$ and $\left\{s_{i}, i \varepsilon I\right\}$ be as in the proof of Proposition 6.2. For each $1 \leqq j \leqq n$ and $x \varepsilon X$, we denote the prime ideal of $L_{x}$ which arises 
from $\mathfrak{p}_{j}$ by $\mathfrak{p}_{j, x}$. The meaning of $\omega$ is the same as in the proof of Proposition 8.3 which has just been completed. We proceed in two steps.

(a) $x \varepsilon Y_{i} \subset V$ for some $1 \leqq j \leqq n$. Select an $i \varepsilon I$ such that $x \varepsilon U_{i}$. We know from Proposition 6.3 that $V$ is the variety of the coherent sheaf $B$ of ideals, where $B_{x}=s_{i}(x) L_{x}$. We also know that $Y_{j}$ is the variety of a coherent sheaf $\mathfrak{a}$ of ideals, where $\mathfrak{a}_{x}=\mathfrak{p}_{i, x}$. It now follows from Lemma 8 on page 7 of [2] that $s_{i}(x) \varepsilon \mathfrak{p}_{j, x}$. The fact that $x \in Y_{j}$ implies that $\mathfrak{p}_{j, x}$ is a nonunit associated prime ideal of $\omega$, whence $s_{i}(x) g=0$ for some nonzero $g \varepsilon F_{x}$. This proves, according to Proposition 3.2, that $x \varepsilon \operatorname{Sup}(\operatorname{ker}(w))$.

(b) $x \varepsilon \operatorname{Sup}(\operatorname{ker}(w))$. Select an $i \varepsilon I$ such that $x \varepsilon U_{i}$. According to Proposition 3.2 , there exists a nonzero $g \varepsilon F_{x}$ with the property that $s_{i}(x) g=0$. Consequently, $s_{i}(x)$ belongs to some nonunit associated prime ideal $\mathfrak{p}_{i, x}$ of $\omega$, for some $1 \leqq j \leqq n$. This means that $x \varepsilon Y_{i}$ and $B_{x} \subset \mathfrak{a}_{x}$, where $\mathfrak{a}$ has the same meaning as above. It follows that there exists an open neighborhood $U$ of $x$ such that $B_{y} \subset a_{y}$ for all $y \varepsilon U$. This implies that $Y_{j} \cap U \subset V \cap U$ and hence that $Y_{j} \cap U \subset V$. The closure relative to $Y_{i}$ of the nonempty, open subset $Y_{j} \cap U$ of the irreducible topological space $Y_{i}$ is equal to $Y_{i}$, and this closure is, of course, contained in the closed subset $V$ of $X$. We have now proved that $x \varepsilon Y_{i} \subset V$.

We refer to the above varieties $Y_{1}, \cdots, Y_{n}$ as the associated varieties of $F$. Since $V \neq X$, we conclude from Propositions 8.3 and 8.4 that Sup (ker $(w)$ ) $\subset$ $V \cap \operatorname{Sup}(T)$, which agrees with Proposition 6.2. It is now easy to construct examples galore where Sup $(\operatorname{ker}(w)) \neq V \cap \operatorname{Sup}(T)$. For instance, let $X$ be the projective plane and $Y$ a straight line in $X$. We will use the sheaf of local rings of $Y$, extended by zero to $X$, as the sheaf $F$. Then $F=T$, whence $Y=$ Sup $(T)$. Furthermore, $Y$ is the only associated variety of $F$. (This follows immediately from the fact that $\mathfrak{M}$ is the $\mathfrak{D}$-module $\mathfrak{o} / \mathfrak{p}$, where $\mathfrak{p}$ is the homogeneous prime ideal of $\mathfrak{v}$ which corresponds to $Y$.) Select some other straight line $V \neq Y$ in $X$, and let $x$ be the point of intersection of $V$ and $Y$. A unique prime divisor $\mathfrak{B}$ of $X$ is centered at $V$, and we will use the unique line class of $X$ which corresponds to $\mathfrak{B}$ as $f$. (Observe that $f=p$.) We choose the nonzero section $s$ of $f(L)$ over $X$ in such a way that $(s)=\mathfrak{P}$. (This can be done since $X$ is normal; see section 5.) Clearly $V \cap \operatorname{Sup}(T)=x$, but Sup $(\operatorname{ker}(w))=\varnothing$. We remark for completeness sake that we now have the exact sequence $0 \rightarrow F \rightarrow f(F) \rightarrow$ coker $(w) \rightarrow 0$ and that, according to Proposition 6.2, Sup $(\operatorname{coker}(w))=x$.

Proposition 8.5. Let $X$ be normal and $k$ infinite. Let $f$ be a regular line class of $X$ whose complete linear system $|f|$ is free of base points. For any algebraic coherent sheaf $F$ of $X$, we can select the nonzero $s \varepsilon S(X, f(L))$ in such a way that $w: F \rightarrow f(F)$ is a monomorphism.

Proof. Let $Y_{1}, \cdots, Y_{n}$ be the associated varieties of $F$, and denote the $k$-linear vector space $S(X, f(L))$ by $S$. If $F=0$, Proposition 8.5 is trivially correct. If $F \neq 0$, select $x_{i} \varepsilon Y_{i}$ for $j=1, \cdots, n$. The nonzero elements $s \varepsilon S$ with the property that $x_{i} \varepsilon \operatorname{Var}(s)$ form, together with the zero element of $S$, a $k$-linear 
subspace $S_{i}$ of $S$; this follows immediately from Proposition 6.1. Since $x_{i}$ is not a base point of $|f|$, each $S_{i} \neq S$. (We use here that the $\lambda$ of Proposition 5.3 is onto, which follows from the normality of $X$.) Hence, because $k$ is infinite, there exists an $s \varepsilon S$ such that $s, S_{j}$ for $j=1, \cdots, n$. This $s$ is necessarily nonzero and $x_{i} \& \operatorname{Var}(s)$ for $j=1, \cdots, n$. It follows from Proposition 8.4 that if this $s$ is used to construct the mapping $w$, then Sup $(\operatorname{ker}(w))=\varnothing$.

We do not know whether it is possible to remove the condition that $k$ is infinite from Proposition 8.5. We observe that we may always use the line class $p$ as the $f$ of this proposition. This is, essentially, what was done in section 81 of [1].

9. The principal theorem. We assume that $X$ is a projective variety which is not necessarily irreducible. The symbols $k, L$ and $L_{0}$ retain their previous meanings. Again, $r$ is the dimension of $X$ and $Z$ is the ring of the rational integers. If $F$ is an algebraic coherent sheaf over $X$, then $\chi(X, F)=\sum(-1)^{t} h^{t}(X, F)$, where $t \geqq 0$, is the Euler-Poincare characteristic of $X$ with values in $F$; as usual, $h^{t}(X, F)=\operatorname{dim}_{k}\left(H^{t}(X, F)\right)$. The principal theorem is the following.

Theorem 9.1. Let $f_{1}, \cdots, f_{n}$ be a finite set of line classes of $X$ and $F$ an algebraic coherent sheaf of $X$. Then $\chi\left(X, f_{1}^{m_{1}} \cdots f_{n}^{m_{n}}(F)\right)$ is a rational polynomial in $m_{1}$, $\cdots, m_{n}$ for all $m_{1}, \cdots, m_{n} \varepsilon Z$. The degree of this polynomial does not exceed $\operatorname{dim}(\operatorname{Sup}(F))$.

Proof. It is obvious that Theorem 9.1 is correct if $\operatorname{dim}(X)=0$. Namely, $X$ is then a discrete topological space, whence $H^{1}\left(X, L_{0}\right)$ consists of only the identity element. (This follows immediately from the observation that the open covering of $X$, which consists of the individual points of $X$, has dimension zero and is a refinement of every open covering of $X$; the corollary in section 20 of [1] must also be used.) It follows that $\chi\left(X, f_{1}^{m_{1}} \cdots f_{n}^{m_{n}}(F)\right)=\chi(X, F)$, which shows that Theorem 9.1 is correct if $\operatorname{dim}(X)=0$.

We now make the induction hypothesis that Theorem 9.1 has been proved for $\operatorname{dim}(X)<r$, and proceed by considering several less and less restrictive special cases.

Case 1. Sup $(F) \subset Y$, where $Y$ is a closed subset of $X$ and $\operatorname{dim}(Y)<r$. Furthermore, $F(Y)$ is an algebraic coherent sheaf of $Y$. (As always, $F(Y)$ denotes the restriction of $F$ to $Y$.) Let $L^{\prime \prime}$ be the sheaf of local rings of $Y$ and $L^{\prime}$ the extension of $L^{\prime \prime}$ by zero to $X$. We know from section 2 that, for each $i=1, \cdots, n$, the line class $f_{i}$ gives rise to a line class $f_{i}^{\prime}$ of $L^{\prime}$ and $f_{i}^{\prime}$ gives rise to a line class $f_{i}^{\prime \prime}$ of $L^{\prime \prime}$. The mapping $f_{i} \rightarrow f_{i}^{\prime \prime}$ is a group homomorphism from $H^{1}\left(X, L_{0}\right)$ into $H^{1}\left(Y, L_{0}^{\prime \prime}\right)$, whence $\left(f_{1}^{m_{1}} \cdots f_{n}^{m_{n}}\right)^{\prime \prime}=\left(f_{1}^{\prime \prime}\right)^{m_{2}} \cdots\left(f_{n}^{\prime \prime}\right)^{m_{n}}$ for all $m_{1}, \cdots, m_{n} \varepsilon Z$. We now immediately conclude from Proposition 2.5 that $\chi\left(X, f_{1}^{m_{1}} \ldots f_{n}^{m_{n}}(F)\right)=$ $\chi\left(Y,\left(f_{1}^{\prime \prime}\right)^{m_{1}} \cdots\left(f_{n}^{\prime \prime}\right)^{m_{n}}(F(Y))\right)$. The induction hypothesis may be applied to $Y$, $f_{1}^{\prime \prime}, \cdots, f_{n}^{\prime \prime}$ and $F(Y)$, which completes the proof in case 1. 
Case 2. $\operatorname{dim}(\operatorname{Sup}(F))<r$. It follows from Proposition 1 of section 2 of [2] that there exists a finite sequence $F=F_{0} \supset F_{1} \supset \cdots \supset F_{a}=0$ of algebraic coherent sheaves of $X$ with the property that $F_{b} / F_{b+1}$ is algebraic coherent over $\operatorname{Sup}(F)$ for $b=0, \cdots, a-1$. We know from Proposition 2.2 that the exact sequence $0 \rightarrow F_{b+1} \rightarrow F_{b} \rightarrow F_{b} / F_{b+1} \rightarrow 0$ gives rise to an exact sequence

$$
0 \rightarrow f_{1}^{m_{1}} \cdots f_{n}^{m_{n}}\left(F_{b+1}\right) \rightarrow f_{1}^{m_{1}} \cdots f_{n}^{m_{n}}\left(F_{b}\right) \rightarrow f_{1}^{m_{1}} \cdots f_{n}^{m_{n}}\left(F_{b} / F_{b+1}\right) \rightarrow 0
$$

from which it follows that $\chi\left(X, f_{1}^{m_{1}} \cdots f_{n}^{m_{n}}\left(F_{b}\right)\right)$ is the difference of the corresponding characteristics for $F_{b+1}$ and $F_{b} / F_{b+1}$; again, $m_{1} \cdots, m_{n} \varepsilon Z$. Hence if Theorem 9.1 is correct for the sheaves $F_{b+1}$ and $F_{b} / F_{b+1}$, it is also correct for the sheaf $F_{b}$. Using Sup $(F)$ as the $Y$ of case 1, we conclude from case 1 that Theorem 9.1 is correct for all the sheaves $F_{b} / F_{b+1}$ and hence, in particular, for $F_{a-1}$. By putting $b$ equal to, successively, $a-2, \cdots, 0$ we conclude that Theorem 9.1 is correct for $F$; case 2 is complete.

Case 3. $\operatorname{dim}\left(\operatorname{Sup}\left(F^{\prime}\right)\right)=r$. In order to complete this case, and thereby the proof of Theorem 9.1, we need a little arithmetic preparation. $Q$ denotes the field of rational numbers and $Z^{n}$ the $n$-fold cartesian product of $Z$ with itself.

Proposition 9.1. Let the function $q\left(z_{1}, \cdots, z_{n}\right): Z^{n} \rightarrow Q$ have the following property. There exists a nonnegative integer $d$ such that $q\left(m_{1}, \cdots, m_{i-1}, z_{i}\right.$, $\left.m_{i+1}, \cdots, m_{n}\right)$ is a rational polynomial in $z_{i}$ of degree at most $d$ for all choices of $m_{1}, \cdots, m_{i-1}, m_{i+1}, \cdots, m_{n} \varepsilon Z$ and of $i=1, \cdots, n$. In this case, $q\left(z_{1}, \cdots\right.$, $z_{n}$ ) is a rational polynomial in $z_{1}, \cdots, z_{n}$. (I.e., $q\left(z_{1}, \cdots, z_{n}\right) \varepsilon Q\left[z_{1}, \cdots, z_{n}\right]$.)

Proof. $(\alpha)$ Let $n=2$. Then

$$
q\left(z_{1}, z_{2}\right)=a_{0}\left(z_{1}\right)+a_{1}\left(z_{1}\right) z_{2}+\cdots+a_{d}\left(z_{1}\right) z_{2}^{d}
$$

where each coefficient is a well defined function $a_{j}\left(z_{1}\right): Z \rightarrow Q$, for $j=0, \cdots, d$. In order to show that each $a_{j}\left(z_{1}\right) \varepsilon Q\left[z_{1}\right]$, select $d+1$ mutually distinct elements $c_{0}, \cdots, c_{d} \varepsilon Z$. We are given that

$$
q\left(z_{1}, c_{i}\right)=a_{0}\left(z_{1}\right)+a_{1}\left(z_{1}\right) c_{j}+\cdots+a_{d}\left(z_{1}\right) c_{j}^{d} \varepsilon Q\left[z_{1}\right]
$$

for $j=0, \cdots, d$. This is a nonsingular, $d+1 \times d+1$, system of linear equations for the coefficients $a_{j}\left(z_{1}\right)$. If we solve it, we see that each $a_{i}\left(z_{1}\right)$ is a linear combination of $q\left(z_{1}, c_{0}\right), \cdots, q\left(z_{1}, c_{d}\right)$ with rational coefficients, whence $a_{i}\left(z_{1}\right) \varepsilon Q\left[z_{1}\right]$ for $j=0, \cdots, d$.

$(\beta)$ Let $n>2$. We make the induction hypothesis that Proposition 9.1 has been proved for functions with less than $n$ variables. As under $(\alpha), q\left(z_{1}, \cdots\right.$, $\left.z_{n}\right)=\sum a_{i}\left(z_{1}, \cdots, z_{n-1}\right) z_{n}^{i}$ where $j=0, \cdots, d$. Each coefficient is a well defined function $a_{j}\left(z_{1}, \cdots, z_{n-1}\right): Z^{n-1} \rightarrow Q$. Again, select $d+1$ mutually distinct elements $c_{0}, \cdots, c_{d} \varepsilon Z$. For each $0 \leqq j \leqq d$, we conclude from the induction hypothesis that $q\left(z_{1}, \cdots, z_{n-1}, c_{j}\right) \varepsilon Q\left[z_{1}, \cdots, z_{n-1}\right]$. We show, as under $(\alpha)$, that each $a_{i}\left(z_{1}, \cdots, z_{n-1}\right)$ is a linear combination of the $q\left(z_{1}, \cdots, z_{n-1}, c_{i}\right)$ 
with rational coefficients, whence $a_{i}\left(z_{1}, \cdots, z_{n-1}\right) \varepsilon Q\left[z_{1}, \cdots, z_{n-1}\right]$ for $j=0$, $\cdots, d$.

We observe that all we used was that $Z$ is an integral domain with at least $d+1$ mutually distinct elements, and that $Q$ is a field which contains $Z$.

We now return to the proof of Theorem 9.1 in case 3 . We split this case up in several, less and less restrictive, subcases. In the remainder of this proof we continue the hypothesis that $\operatorname{dim}(\operatorname{Sup}(F))=r$.

Case 3a. $X$ is irreducible, $n=1$ and $f_{1}$ is regular. We write $f$ for $f_{1}$ and consider the $L$-homomorphism $w: F \rightarrow f\left(F^{\prime}\right)$ of section 6. We know from Proposition 2.2 that the exact sequence

$$
0 \rightarrow \operatorname{ker}(w) \rightarrow F \rightarrow f(F) \rightarrow \operatorname{coker}(w) \rightarrow 0
$$

gives rise to an exact sequence

$$
0 \rightarrow f^{m}(\operatorname{ker}(w)) \rightarrow f^{m}(F) \rightarrow f^{m+1}(F) \rightarrow f^{m}(\operatorname{coker}(w)) \rightarrow 0
$$

for all $m \varepsilon Z$. This implies that

$$
\chi\left(X, f^{m+1}\left(F^{\prime}\right)\right)-\chi\left(X, f^{m}\left(F^{\prime}\right)\right)=\chi\left(X, f^{m}(\operatorname{coker}(w))\right)-\chi\left(X, f^{m}(\operatorname{ker}(w))\right) .
$$

It follows from Proposition 6.2 that $\operatorname{dim}(\operatorname{Sup}(\operatorname{coker}(w)))<r$ and that $\operatorname{dim}$ (Sup $(\operatorname{ker}(w)))<r$, whence we conclude from case 2 that "the first difference" $\chi\left(X, f^{m+1}(F)\right)-\chi\left(X, f^{m}(F)\right)$ of the function $\chi\left(X, f^{m}(F)\right)$ is a rational polynomial in $m$ whose degree does not exceed $r-1$. Hence $\chi\left(X, f^{m}(F)\right)$ is a rational polynomial in $m$ whose degree does not exceed $r$; case $3 a$ is complete.

Case $3 b . X$ is irreducible and $f_{1}, \cdots, f_{n}$ are regular. We put $\chi\left(X, f_{1}^{z_{1}} \cdots\right.$ $\left.f_{n}^{z_{n}}(F)\right)=q\left(z_{1}, \cdots, z_{n}\right)$ and study the well defined function $q\left(z_{1}, \cdots, z_{n}\right)$ : $Z^{n} \rightarrow Z$; here $Z^{n}$ has the same meaning as in Proposition 9.1. We assert that $q\left(m_{1}, \cdots, m_{i-1}, z_{i}, m_{i+1}, \cdots, m_{n}\right)$ is a rational polynomial in $z_{i}$ of degree at most $r$ for all choices of $m_{1}, \cdots, m_{i-1}, m_{i+1}, \cdots, m_{n} \varepsilon Z$ and of $i=1, \cdots, n$. Namely, according to Proposition 2.2, the algebraic coherent sheaf

$$
F^{\prime}=f_{1}^{m_{1}} \cdots f_{i-1}^{m_{i}-1} f_{i+1}^{m_{i+1}} \cdots f_{n}^{m_{n}}(F)
$$

of $X$ is such that

$$
f_{i}^{z_{i} i}\left(F^{\prime}\right)=f_{1}^{m_{1}} \cdots f_{i-1}^{m_{i-1}} f_{i}^{z_{i}} f_{i+1}^{m_{i+1}} \cdots f_{n}^{m_{n}}(F) .
$$

This shows that $q\left(m_{1}, \cdots, m_{i-1}, z_{i}, m_{i+1}, \cdots, m_{n}\right)=\chi\left(X, f_{i}^{z_{i}}\left(F^{\prime}\right)\right)$, and we conclude from case 3 a that $q\left(m_{1}, \cdots, m_{i-1}, z_{i}, m_{i+1}, \cdots, m_{n}\right)$ is as asserted. (Observe that $F^{\prime}$ and $F$ have the same support since they are locally isomorphic.) We now infer from Proposition 9.1 that $q\left(z_{1}, \cdots, z_{n}\right) \varepsilon Q\left[z_{1}, \cdots, z_{n}\right]$, and all that remains to be shown is that the degree of this polynomial does not exceed $r$. If $q\left(z_{1}, \cdots, z_{n}\right)=0$, there is nothing to be proved. If $q\left(z_{1}, \cdots, z_{n}\right) \neq 0$, let $d$ be its degree and select nonnegative integers $m_{1}, \cdots, m_{n} \varepsilon Z$ such that the degree of the polynomial $q\left(m_{1} z, \cdots, m_{n} z\right) \varepsilon Q[z]$ is still $d$. If $f$ denotes the regular line class $f_{1}^{m_{1}} \cdots f_{n}^{m_{n}}$ of $X$, we see that $q\left(m_{1} z, \cdots, m_{n} z\right)=\chi\left(X, f^{z}(F)\right)$, and we 
conclude from case $3 \mathrm{a}$ that the degree of $q\left(m_{1} z, \cdots, m_{n} z\right)$ is at most $r$; case $3 \mathrm{~b}$ is complete.

Case 3c. $X$ is irreducible. According to Corollary 8.1, we can express $f_{i}=\prod g_{i j}^{t_{i j}}$, where the product is with respect to $j=1, \cdots, s(i)$ and where all the $g_{i j}$ are regular line classes of $X$; of course, $t_{i j} \varepsilon Z$ and $i=1, \cdots, n$. Hence $f_{1}^{m_{1}} \cdots f_{n}^{m_{n}}=\prod g_{i j}^{t_{i j} m_{i}}$, where the product is now with respect to both $i$ and $j$. We know from case $3 \mathrm{~b}$ that $\chi\left(X, g_{11}^{z_{11}} \cdots g_{n s(n)}^{z_{n s}(n)}(F)\right)$ is a rational polynomial $q^{\prime}\left(z_{11}, \cdots, z_{n s(n)}\right)$ in the $s(1)+\cdots+s(n)$ variables $z_{i j}$ of degree at most $r$. If we substitute $t_{i j} z_{i}$ for $z_{i j}$ in $q^{\prime}\left(z_{11}, \cdots, z_{n s(n)}\right)$, we obtain a rational polynomial $q\left(z_{1}, \cdots, z_{n}\right)$ of degree at most $r$ in the variables $z_{1}, \cdots, z_{n}$. Since $q\left(z_{1}, \cdots\right.$, $\left.z_{n}\right)=\chi\left(X, f_{1}^{z_{1}} \cdots f_{n}^{z_{n}}\left(F^{\prime}\right)\right)$, we are done with case $3 \mathrm{c}$.

Case 3d. All restrictions are dropped, except that $\operatorname{dim}(\operatorname{Sup}(F))=r$. Let $X=$ $X_{1} \cup \ldots \cup X_{t}$ be the decomposition of $X$ into its irreducible components. We know from section 3 of [2] that there exist algebraic coherent sheaves $F_{1}, \cdots$, $F_{t}$ of $X$ which have the following two properties: (1) Sup $\left(F_{i}\right) \subset X_{i}$ and the restriction $F_{i}^{\prime}$ of $F_{i}$ to $X_{i}$ is algebraic coherent over $X_{i}$ for $j=1, \cdots, t$. (2) If $G$ is the direct sum of $F_{1}, \cdots, F_{t}$, there exists an $L$-homomorphism $v: F \rightarrow G$ such that $\operatorname{dim}(\operatorname{Sup}(\operatorname{ker}(v)))<r$ and $\operatorname{dim}(\operatorname{Sup}($ coker $(v)))<r$. We conclude again from Proposition 2.2 that we have an exact sequence

$0 \rightarrow f_{1}^{m_{1}} \cdots f_{n}^{m_{n}}(\operatorname{ker}(v)) \rightarrow f_{1}^{m_{1}} \cdots f_{n}^{m_{n}}\left(F^{\prime}\right)$

$$
\rightarrow f_{1}^{m_{1}} \cdots f_{n}^{m_{n}}(G) \rightarrow f_{1}^{m_{1}} \cdots f_{n}^{m_{n}}(\operatorname{coker}(v)) \rightarrow 0
$$

for all choices of $m_{1}, \cdots, m_{n} \varepsilon Z$. Since $\chi\left(X, f_{1}^{m_{1}} \cdots f_{n}^{m_{n}}(\operatorname{ker}(v))\right)$ and $\chi(X$, $f_{1}^{m_{1}} \cdots f_{n}^{m_{n}}($ coker $\left.(v))\right)$ are rational polynomials in $m_{1}, \cdots, m_{n}$ of degrees at most $r-1$ according to case 2 , all that remains to be shown is that $\chi\left(X, f_{1}^{m_{1}} \cdots f_{n}^{m_{n}}(G)\right)$ is a rational polynomial in $m_{1}, \cdots, m_{n}$ of degree at most $r$. Accordingly, we denote the sheaf of local rings of $X_{j}$ by $L_{j}$ and the line class of $X_{i}$ which arises from $f_{i}$ by $f_{i j}$. We have seen in Proposition 2.5 that

$$
\chi\left(X, f_{1}^{m_{1}} \cdots f_{n}^{m_{n}}\left(F_{j}\right)\right)=\chi\left(X_{j}, f_{1 j}^{m_{1}} \cdots f_{n j}^{m_{n}}\left(F_{i}^{\prime}\right)\right),
$$

whence we conclude from case $3 \mathrm{c}$ that $\chi\left(X, f_{1}^{m_{1}} \cdots f_{n}^{m_{n}}\left(F_{i}\right)\right)$ is a polynomial in $m_{1}, \cdots, m_{n}$ of degree at most $r$. We finally observe that $\chi\left(X, f_{1}^{m_{1}} \cdots f_{n}^{m_{n}}(G)\right)=$ $\sum \chi\left(X, f_{1}^{m_{1}} \cdots f_{n}^{m_{n}}\left(F_{j}\right)\right)$ where $j=1, \cdots, t$, and we are completely done.

The special case of Theorem 9.1 , where $n=1$ and where $f_{1}$ is the line class $p$ of section 8 , was discussed in section 81 of [1]. The reason why in this case the degree of the polynomial $\chi\left(X, p^{m}(F)\right)$ is precisely equal to $\operatorname{dim}(\operatorname{Sup}(F))$ will be analyzed in our paper Polynomials associated with divisors. See also example 10.1 .

10. Examples. We show by means of examples that, in two respects, Theorem 9.1 is as strong as possible. 
Example 10.1. Let $k$ be the field of complex numbers and $X$ a nonsingular quadric, imbedded in 3-dimensional complex projective space. Hence $r=2$. Let $Y_{1}$ and $Y_{2}$ be two straight lines of $X$ such that $Y_{1} \cap Y_{2}=\varnothing$. We choose for the $F$ of Theorem 9.1 the extension by zero of the sheaf $L_{1}$ of local rings of $Y_{1}$. It follows that $\operatorname{Sup}(F)=Y_{1}$ and that $F$ is equal to its torsion sheaf $T$. Since $X$ is normal, a unique prime divisor $\mathfrak{B}$ of $X$ is centered at $Y_{2}$, and we know from section 5 that a unique, regular line class $f$ of $X$ corresponds to $\mathfrak{P}$. We assert that $\chi\left(X, f^{m}(F)\right)=1$ for all $m \varepsilon Z$. Accordingly, consider the $L$ homomorphism $w: F \rightarrow f(F)$ which is associated with $F, f$ and $\mathfrak{B}$. We know from Proposition 6.2 that

$$
\operatorname{Sup}(\operatorname{ker}(w)) \subset \operatorname{Var}(\mathfrak{B}) \cap \operatorname{Sup}(T)=Y_{2} \cap Y_{1}=\phi
$$

and that

$$
\operatorname{Sup}(\operatorname{coker}(w))=\operatorname{Var}(\mathfrak{B}) \cap \operatorname{Sup}(F)=Y_{2} \cap Y_{1}=\phi,
$$

which shows that $w$ is an isomorphism. Proposition 2.2 now tells us that $F \simeq f^{m}(F)$ for all $m \varepsilon Z$, whence $\chi\left(X, f^{m}(F)\right)=\chi(X, F)$ for all $m \varepsilon Z$. Finally, $\chi(X, F)=\chi\left(Y_{1}, L_{1}\right)=1-g$ where $g$ is the genus of the straight line $Y_{1}$. Since $g=0$, the assertion is proved. This then gives us an example where the degree of the polynomial $\chi\left(X, f^{m}(F)\right.$ ) is less than $\operatorname{dim}(\operatorname{Sup}(F))$, even though $k$ is the field of complex numbers, $X$ is nonsingular and $|f|$ is without base points. Of course, the example works because $Y_{1} \cap Y_{2}=\varnothing$, and this situation will be further analyzed in Polynomials associated with divisors.

Example 10.2. Let $k$ be the field of complex numbers and $X$ a quadratic cone, imbedded in 3-dimensional complex projective space. Again, $r=2$ and $X$ is irreducible, but $X$ is now singular; however, $X$ is normal because it is a surface in 3-space without 1-dimensional singularities. Let $Y$ be a straight line on $X$ and let $\mathfrak{P}$ be the unique prime divisor of $X$ which is centered at $Y$. The divisor $D=\mathfrak{B}$ is not locally linearly equivalent to zero, and we will show that Theorem 9.1 fails to hold for $D$. (This failure of Theorem 9.1 for $D$ is, of course, in itself complete proof that $D$ is not locally linearly equivalent to zero.) Precisely, the sheaf $L(m D)$, where $L$ is the sheaf of local rings of $X$, is well defined for all $m \varepsilon Z$ (see, for instance, [6]), and we show that there exists no rational polynomial $a_{0}+a_{1} m+a_{2} m^{2}$ which is equal to $\chi(X, L(m D))$ for all $m \varepsilon Z$. If such a polynomial existed, then

$$
a_{0}+2 a_{1} n+4 a_{2} n^{2}=\chi(X, L(2 n D))
$$

for all $n \varepsilon Z$. The divisor $2 D$ is cut out on $X$ by a tangent plane of $X$, whence $L(2 n D) \simeq p^{n}(L)$ for all $n \varepsilon Z$; here $p$ is the line class of $X$, defined in section 8 . It is well known (and will also be derived in Polynomials associated with divisors) that $\chi\left(X, p^{n}(L)\right)=1+2 n+n^{2}$ for all $n \varepsilon Z$, and this would imply that $a_{0}=$ $a_{1}=1$ and $a_{2}=\frac{1}{4}$. We would then come to the conclusion that $\chi(X, L(D))=\frac{9}{4}$, which is an absurdity. 
11. A lemma concerning $H^{r}\left(X, f^{m}(F)\right)$. Theorem 9.1 makes a statement about the whole alternating sum $\chi\left(X, f_{1}^{m_{1}} \cdots f_{n}^{m_{n}}(F)\right)$. For theorems of the Riemann-Roch type one wants information about just the first term $h^{0}\left(X, f_{1}^{m_{1}} \ldots\right.$ $\left.f_{n}^{m_{n}}(F)\right)$. In general one may say that one of the great problems of present day algebraic geometry is to obtain information about $h^{t}\left(X, f_{1}^{m_{1}} \cdots f_{n}^{m_{n}}(F)\right)$ as a function of $m_{1}, \cdots, m_{n}$, for some fixed $t$. Lemma 11.1 gives information of this kind.

We assume for the remainder of this section that $X$ is a complete, irreducible algebraic variety of dimension $r$ and with sheaf of local rings $L$; it is not necessary that $X$ be projective. Let the algebraic coherent sheaf $F$ of $X$ and the regular line class $f$ of $X$ be related in such a way that the $L$-homomorphism $w: F \rightarrow f(F)$ of section 6 is a monomorphism for proper choice of $s \varepsilon S(X, f(L))$.

Lemma 11.1. $h^{r}\left(X, f^{m}(F)\right) \geqq h^{r}\left(X, f^{n}(F)\right)$ for all $m \leqq n \varepsilon Z$. Consequently, there exists an $m_{0} \varepsilon Z$ such that, for all $m \geqq m_{0}, h^{r}\left(X, f^{m}(F)\right)$ is constant.

Proof. The exact sequence

$$
0 \rightarrow F \rightarrow f(F) \rightarrow \operatorname{coker}(w) \rightarrow 0
$$

gives rise to the exact sequence

$$
0 \rightarrow f^{m}(F) \rightarrow f^{m+1}(F) \rightarrow f^{m}(\operatorname{coker}(w)) \rightarrow 0
$$

and hence to the exact sequence

$$
H^{r}\left(X, f^{m}(F)\right) \rightarrow H^{r}\left(X, f^{m+1}(F)\right) \rightarrow 0 ;
$$

here we use that $\operatorname{dim}\left(\operatorname{Sup}\left(f^{m}(\operatorname{coker}(w))\right)\right)<r$ according to Proposition 6.2, whence $H^{r}\left(X, f^{m}\right.$ (coker $\left.\left.(w)\right)\right)=0$ according to Lemma 9 of section 3 of [2]. It now follows that, for all $m \varepsilon Z, h^{r}\left(X, f^{m}(F)\right) \geqq h^{r}\left(X, f^{m+1}(F)\right)$, and Lemma 11.1 is an immediate consequence of this inequality.

There are many instances where the conditions of Lemma 11.1 are satisfied. For example, if the torsion sheaf of $F$ is zero, we see from Proposition 6.2 that Lemma 11.1 holds for all the regular line classes $f$ of $X$. Since $L$ is free of torsion, this proves the following corollary.

Corollary 11.1. Let $X$ be normal and $D$ a nonnegative divisor of $X$ which is locally linearly equivalent to zero. Then $h^{r}(X, L(m D)) \geqq h^{r}(X, L(n D))$ for all $m \leqq n \varepsilon Z$. There exists an $m_{0} \varepsilon Z$ such that, for all $m \geqq m_{0}, h^{r}(X, L(m D))$ is constant.

We assume for the remainder of this section that our $X$ is, furthermore, a projective variety. If $X$ and its regular line class $f$ satisfy the conditions of Proposition 8.5, we may apply Lemma 11.1 for all the algebraic coherent sheaves $F$ of $X$. Whenever Lemma 11.1 may be applied it shows, together with Theorem 9.1 , that the alternating sum $\sum(-1)^{t} h^{t}\left(X, f^{m}(F)\right)$, where $t=0, \cdots, r-1$, is a rational polynomial in $m$ for all large enough $m \varepsilon Z$, whose degree is at most 
equal to $\operatorname{dim}(\operatorname{Sup}(F)$ ). In case $X$ is nonsingular, Corollary 11.1 follows also from the duality theorem which tells us that then $h^{r}(X, L(m D))=0$ for positive $D$ and large enough $m$.

\section{References}

[1] SerRe, J. P.: Faisceaux algébriques coherents, Annals of Mathematics, 61 (1955), pp. 197278.

[2] Serre, J. P.: Sur la cohomologie des variétés algébriques, Journ. de Math. pures et appliquées, 36 (1957), pp. 1-16.

[3] Grothendimck, A.: A general theory of fibre spaces with structure sheaf, mimeographed notes, Univ. of Kansas (1955).

[4] Hirzebruch, F.: Neue topologische Methoden in der Algebraischen Geometrie, Ergebnisse, Vol. 9 (1956).

[5] Kodarra, K.: Characteristic linear systems of complete continuous systems, Mimeographed notes, Princeton Univ. (1954).

[6] SNappen, E.: Cohomology theory and algebraic correspondences, Memoirs of the Amer. Math. Soc., No. 33 (1959).

[7] Snapper, E.: Cohomology groups and genera of higher-dimensional fields, Memoirs of the Amer. Math. Soc., No. 28 (1957).

[8] Snapper, E.: Higher-dimensional field theory II, Compositio Mathematica, 13 (1956), pp. 16-38.

[9] Cartan, H. \& Eilenberg, S.: Homological algebra, Princeton Univ. Press (1956).

[10] Grothendieck, A.: Sur les faisceaux algébriques et les faisceaux analytiques cohérents, Séminaire H. Cartan, E.N.S., exposé No. 2 (1956-1957), pp. 2-01-2-16.

[11] Zariski, O.: Algebraic sheaf theory, Bull. of the Amer. Math. Soc., 62, No. 2 (1956), pp. 117-141.

Indiana University

Bloomington, Indiana 